\title{
Método de Chen-Stein e o Modelo de Ehrenfest
}

José Domingo Restrepo Alvarez

DISSERTAÇÃO APRESENTADA

$\mathrm{AO}$

INSTITUTO DE MATEMÁTICA E ESTATÍSTICA

DA

UNIVERSIDADE DE SÃO PAULO

PARA OBTENÇÃO DO GRAU

DE

MESTRE EM ESTATÍSTICA

Área de Concentração: Probabilidade

Orientador: Prof. Dr. Adilson Simonis

Apoio financeiro do $\mathrm{CNPq}$

-São Paulo, julho de 2000- 
MÉTODO DE CHEN-STEIN E O MODELO DE EHRENFEST

Este exemplar corresponde à redação final da dissertação devidamente corrigida e defendida por José Domingo Restrepo Alvarez e aprovada pela comissão julgadora.

São Paulo, 20 de julho de 2000.

Banca Examinadora:

- Prof. Dr. Adilson Simonis (Presidente) - IME - USP

- Prof. Dr. Luiz Renato Gonçalves Fontes - IME - USP

- Prof. Dr. Yoshiharu Kohayakawa - IME - USP 
Em memória de meu pai Luis Ovidio. A minha mãe Eloisa e meus irmãos, pelo amor, compreensão, apoio e sábios conselhos. 


\section{AGRADECIMENTOS}

Agradeço a meu orientador Prof. Adilson Simonis, pela sugestão do tema, pela dedicação, compreensão e paciência durante a orientação desta dissertação.

Aos professores, Cláudia Peixoto, Claudio Possani, Antônio Luiz Pereira e Saulo Barros, pelas importantes discussões e sugestões.

Aos meus amigos, Jairo, Sandra e Gladys, pelo incentivo, amizade, alegria e por estarem sempre presentes em todos os momentos.

Aos meus amigos e compatriotas, Hernan e Paola. A minha amiga Adrilayne, pela ajuda prestada em todos os momentos e pela amizade sincera desde minha chegada a este país.

Mais ainda, a todos os meus amigos do Instituto e outros, cujos nomes aqui não escrevo, pela compreensão em relação à presente omissão.

Ao pessoal da biblioteca do Instituto, pela apoio e amizade incomuns. 


\section{SUMÁRIO}

O objetivo deste trabalho é estudar o método de Chen-Stein o qual determina um limite superior para a velocidade de convergência em distribuição de somas de variáveis aleatórias dependentes Bernoulli à distribuição Poisson. Inicialmente o método foi introduzido por Charles Stein (1970) no contexto do Teorema Central do Limite e a seguir Louis Chen (1975) adaptou essas idéias à distribuição Poisson. Apresentamos e exemplificamos aqui todos os detalhes do principal resultado obtido por Chen. Além disso, aplicamos o método para o modelo de Ehrenfest no contexto do passeio aleatório no hipercubo.

\section{ABSTRACT}

The study of the Chen-Stein method is the main subject of this work which give us an upper bound to the velocity of convergence of a sum of dependent Bernoulli trials to the Poisson distribution. Inicially this method was proposed by Charles Stein (1970) in the context of Central Limit Theorem and after Louis Chen (1975) adapted it to the Poisson distribution. We give here all details with examples about of the Chen's results. Moreover, we also apply this to the Ehrenfest model corresponding a random walk on the hypercube. 


\section{ÍNDICE}

INTRODUÇÃO

1 MÉTODO DE CHEN-STEIN 3

2 ALGUMAS APLICAÇÕES 37

3 MODELO DE EHRENFEST

$\begin{array}{ll}\text { BIBLIOGRAFIA } & 48\end{array}$ 


\section{INTRODUÇÃO}

Em 1970, Charles Stein [15] introduziu, no contexto do Teorema Central do Limite, um método para controlar o erro na aproximação da distribuição de somas de variáveis aleatórias dependentes. A técnica usada foi original e não utilizou métodos de Fourier; em seu lugar aparece um operador diferencial que caracteriza a variável aleatória com lei Normal padrão. Em 1975, Louis Chen [16] adaptou idéias de Stein à distribuição Poisson através de um operador diferenças.

Chen verificou que uma condição necessária e suficiente, para uma variável aleatória ter distribuição Poisson, é que o valor da esperança matemática, quando aplicada àquele operador diferenças nessa variável aleatória, seja zero. O resultado é discutido em diferentes situações (ver: [2], [3], [4], [5], [6], [7] e [14]).

No primeiro capítulo, o Teorema 1.1 nos dará condições necessárias e suficientes para que uma variável aleatória seja Poisson, e no Teorema 1.2, através da distância em variação total entre as leis determinaremos uma cota para a velocidade de convergência em distribuição à Poisson. Isto é, para um conjunto $\Gamma$ enumerável e para cada $\alpha \in \Gamma$, seja $X_{\alpha}$ variável aleatória Bernoulli com $p_{\alpha}=\mathbb{P}\left(X_{\alpha}=1\right)=1-\mathbb{P}\left(X_{\alpha}=0\right)$. Defina $W=\sum_{\alpha \in \Gamma} X_{\alpha}$ e seja $Z$ variável aleatória com lei Poisson de parâmetro $\lambda>0$. Temos que a distância em variação total entre as leis de $W$ e $Z$, que denotaremos $d_{V T}(W, Z)$, satisfaz a seguinte desigualdade

$$
d_{V T}(W, Z) \leq\left(b_{1}+b_{2}\right) \frac{1-e^{-\lambda}}{\lambda}+b_{3} \min \left(1, c_{1} \lambda^{-\frac{1}{2}}\right)
$$

para algum $c_{1}>0$ e onde

$$
b_{1}=\sum_{\alpha \in \Gamma} \sum_{\beta \in B_{\alpha}} \mathbb{E}\left(X_{\alpha}\right) \mathbb{E}\left(X_{\beta}\right),
$$




$$
b_{2}=\sum_{\alpha \in \Gamma} \sum_{\substack{\beta \in B_{\alpha} \\ \beta \neq \alpha}} \mathbb{E}\left(X_{\alpha} X_{\beta}\right)
$$

e

$$
b_{3}=\sum_{\alpha \in \Gamma} \mathbb{E}\left|\mathbb{E}\left(X_{\alpha}-p_{\alpha} / \sum_{\beta \in \Gamma-B_{\alpha}} X_{\beta}\right)\right|
$$

com $B_{\alpha}$ algum subconjunto em $\Gamma$ tal que $\alpha \in B_{\alpha}$. Aqui escrevemos todos os detalhes desse resultado.

No capítulo 2, apresentamos dois exemplos, a saber, o de Rinott e o dos aniversários. No capítulo 3 é estudado o modelo de Ehrenfest de bolas e urnas, tratado no contexto do passeio aleatório no hipercubo. Para esse modelo apresentamos uma cota superior na distância em variação total para a aproximação à Poisson, onde a variável de interesse $W$ é o número de vezes que o passeio aleatório visita o origem, ou na forma de bolas e urnas, a variável aleatória de estudo é o número de vezes que uma urna previamente determinada fica vazia. Nosso resultado para este modelo foi que

$$
d_{V T}(W, Z) \leq \frac{c_{2} t}{N}
$$

onde $Z$ é Poisson, $N$ o número de bolas (ou a dimensão do hipercubo), $t$ o valor médio de $W$ e $c_{2}>0$ uma constante independente de $t$ e $N$. 


\section{Capítulo 1}

\section{MÉTODO DE CHEN-STEIN}

Neste capítulo apresentaremos em detalhes a técnica desenvolvida por Louis Chen em 1975, a qual fornece uma cota para a velocidade de convergência em distribuição de uma variável aleatória à lei Poisson.

O método baseou-se no trabalho de Charles Stein, em 1970, que forneceu uma cota para a velocidade de convergência em distribuição de uma variável aleatória à Normal. Stein considerou o operador diferencial

$$
(L f)(x)=f^{\prime}(x)-x f(x),
$$

com $f: \mathbb{R} \rightarrow \mathbb{R}$ diferenciável. Para $Z$ variável aleatória com lei Normal padrão, ele observou que $\mathbb{E}[(L f)(Z)]=0$ para toda função $f$ diferenciável e limitada, onde $\mathbb{E}$ denota a esperança matemática. De fato

$$
\begin{aligned}
\mathbb{E}[(L f)(Z)] & =\mathbb{E}\left[f^{\prime}(Z)-Z f(Z)\right] \\
& =\int_{-\infty}^{+\infty} \frac{e^{-\frac{1}{2} z^{2}}}{\sqrt{2 \pi}} f^{\prime}(z) d z-\int_{-\infty}^{+\infty} \frac{z e^{-\frac{1}{2} z^{2}}}{\sqrt{2 \pi}} f(z) d z
\end{aligned}
$$

Integrando por partes a primeira integral temos que

$$
\begin{aligned}
\int_{-\infty}^{+\infty} \frac{e^{-\frac{1}{2} z^{2}}}{\sqrt{2 \pi}} f^{\prime}(z) d z & =\left.\frac{e^{-\frac{1}{2} z^{2}}}{\sqrt{2 \pi}} f(z)\right|_{-\infty} ^{+\infty}+\int_{-\infty}^{+\infty} \frac{z e^{-\frac{1}{2} z^{2}}}{\sqrt{2 \pi}} f(z) d z \\
& =\int_{-\infty}^{+\infty} \frac{z e^{-\frac{1}{2} z^{2}}}{\sqrt{2 \pi}} f(z) d z
\end{aligned}
$$


Ainda, foi provado que a esperança acima é zero para toda $f$ diferenciável e limitada somente se $Z$ tiver distribuição Normal padrão.

No contexto da distribuição Poisson, Chen considerou o seguinte operador diferenças

$$
(T f)(x)=\lambda f(x+1)-x f(x)
$$

$\operatorname{com} f: \mathbb{Z}_{+}=\{0,1, \ldots\} \rightarrow \mathbb{R}$ e provou que $\mathbb{E}[(T f)(Z)]=0$ para toda $f$ limitada se, e somente se, $Z$ tiver distribuição Poisson de parâmetro $\lambda>0$. Agora faremos uma exposição do método de Chen. Inicialmente consideremos a seguinte definição.

Definição 1.1 Seja $(\Omega, \mathcal{F})$ um espaço mensurável. A distância em variação total, $d_{V T}$, entre duas medidas de probabilidade $\nu$ e $\mu$ em $(\Omega, \mathcal{F})$ é definida por:

$$
d_{V T}(\nu, \mu)=\sup _{A \in \mathcal{F}}|\nu(A)-\mu(A)|
$$

Lema 1.1 A $d_{V T}$ para medidas de probabilidade em $\left(\mathbb{Z}_{+}, \mathcal{P}\left(\mathbb{Z}_{+}\right)\right)$, onde $\mathcal{P}\left(\mathbb{Z}_{+}\right)$é o conjunto das partes de $\mathbb{Z}_{+}$, é dada por

$$
d_{V T}(\nu, \mu)=\sup _{A \subset \mathbb{Z}_{+}}|\nu(A)-\mu(A)|=\frac{1}{2} \sum_{j \geq 0}|\nu(j)-\mu(j)| .
$$

Prova: Como $\nu$ e $\mu$ são medidas de probabilidade, temos para todo $A \subset \mathbb{Z}_{+}$que

$$
1=\nu(A)+\nu\left(A^{c}\right)=\mu(A)+\mu\left(A^{c}\right)
$$

onde $A^{c}$ é o conjunto complementar de $A$. Assim,

$$
\nu(A)-\mu(A)=\mu\left(A^{c}\right)-\nu\left(A^{c}\right) .
$$

Definindo $V=\left\{j \in \mathbb{Z}_{+}: \nu(j) \geq \mu(j)\right\}$ segue que

$$
d_{V T}(\nu, \mu) \geq \nu(V)-\mu(V)=\frac{1}{2} \sum_{j \geq 0}|\nu(j)-\mu(j)|,
$$

$\mathrm{e}$

$$
\begin{aligned}
|\nu(A)-\mu(A)|= & \frac{1}{2} \mid \nu(A \cap V)-\mu(A \cap V)+\nu\left(A \cap V^{c}\right)-\mu\left(A \cap V^{c}\right) \\
& +\mu\left(A^{c} \cap V\right)-\nu\left(A^{c} \cap V\right)+\mu\left(A^{c} \cap V^{c}\right)-\nu\left(A^{c} \cap V^{c}\right) \mid
\end{aligned}
$$




$$
\begin{aligned}
\leq & \frac{1}{2}\left\{|\nu(A \cap V)-\mu(A \cap V)|+\left|\nu\left(A \cap V^{c}\right)-\mu\left(A \cap V^{c}\right)\right|\right. \\
& \left.+\left|\mu\left(A^{c} \cap V\right)-\nu\left(A^{c} \cap V\right)\right|+\left|\mu\left(A^{c} \cap V^{c}\right)-\nu\left(A^{c} \cap V^{c}\right)\right|\right\} \\
= & \frac{1}{2}\left\{\nu(A \cap V)-\mu(A \cap V)+\mu\left(A \cap V^{c}\right)-\nu\left(A \cap V^{c}\right)\right. \\
& \left.+\nu\left(A^{c} \cap V\right)-\mu\left(A^{c} \cap V\right)+\mu\left(A^{c} \cap V^{c}\right)-\nu\left(A^{c} \cap V^{c}\right)\right\} \\
= & \frac{1}{2}\left\{\nu(V)-\mu(V)+\mu\left(V^{c}\right)-\nu\left(V^{c}\right)\right\} \\
= & \nu(V)-\mu(V) .
\end{aligned}
$$

No que segue iremos denotar a distribuição Poisson com parâmetro $\lambda>0$ por $Z \sim P(\lambda)$ e convencionaremos que $0 !=1$.

A equação $($ em $f), \lambda f(x+1)-x f(x)=h(x)-\mathbb{E}[h(Z)], \operatorname{com} f: \mathbb{Z}_{+} \rightarrow \mathbb{R}$, $Z \sim P(\lambda), x \in \mathbb{Z}_{+}$e $h$ uma função real limitada definida nos inteiros não negativos é utilizada para obter uma estimativa da distância em variação total entre a distribuição da soma de variáveis aleatórias Bernoulli e a distribuição Poisson. Primeiro vamos determinar a solução da equação acima.

Lema 1.2 Para $h: \mathbb{Z}_{+} \rightarrow \mathbb{R}$ limitada fixa e $Z \sim P(\lambda)$, a equação (de ChenStein)

$$
\lambda f(w+1)-w f(w)=h(w)-\mathbb{E}[h(Z)], \quad \text { para } w \geq 0
$$

tem como solução $f(0)$ arbitrário e para $w \geq 1$

$$
f(w)=\sum_{l=0}^{w-1} \frac{(w-1) !}{l !} \lambda^{l-w}\{h(l)-\mathbb{E}[h(Z)]\} .
$$

Prova: O valor de $f(0)$ é irrelevante para $f(w), w \geq 1$ pois $0 f(0)=0$. Seja então $f(0)=0$. Para $w=0$ temos que $\lambda f(1)-0=h(0)-\mathbb{E}[h(Z)]$, isto é,

$$
f(1)=\frac{h(0)-\mathbb{E}[h(Z)]}{\lambda}
$$

e portanto a igualdade (1.2) vale para $w=1$. 
Suponha a igualdade (1.2) válida para $w>1$ arbitrário, então

$$
f(w+1)=\frac{h(w)-\mathbb{E}[h(Z)]+w f(w)}{\lambda}
$$

e usando a hipótese de indução em $w \in \mathbb{Z}_{+}$temos que

$$
\begin{aligned}
f(w+1) & =\frac{h(w)-\mathbb{E}[h(Z)]+w \sum_{l=0}^{w-1} \frac{(w-1) !}{l !} \lambda^{l-w}\{h(l)-\mathbb{E}[h(Z)]\}}{\lambda} \\
& =\frac{h(w)-\mathbb{E}[h(Z)]}{\lambda}+\sum_{l=0}^{w-1} \frac{w !}{l !} \lambda^{l-w-1}\{h(l)-\mathbb{E}[h(Z)]\} \\
& =\sum_{l=0}^{w} \frac{w !}{l !} \lambda^{l-w-1}\{h(l)-\mathbb{E}[h(Z)]\} .
\end{aligned}
$$

Corolário 1.1 Para $h: \mathbb{Z}_{+} \rightarrow \mathbb{R}$ limitada e $Z \sim P(\lambda)$ temos que

$$
f(w)=-\sum_{l=w}^{\infty} \frac{(w-1) !}{l !} \lambda^{l-w}\{h(l)-\mathbb{E}[h(Z)]\} .
$$

Prova: Como $h$ é limitada, isto é, existe $c>0$ tal que $|h(w)| \leq c$ para todo $w \in \mathbb{Z}_{+}$, temos que $\mathbb{E}|h(W)|<\infty$. Portanto,

$$
\begin{aligned}
\sum_{l=0}^{\infty} \frac{(w-1) !}{l !} \lambda^{l-w}\{h(l)-\mathbb{E}[h(Z)]\} & =\frac{(w-1) ! e^{\lambda}}{\lambda^{w}} \sum_{l=0}^{\infty} \frac{e^{-\lambda} \lambda^{l}}{l !}\{h(l)-\mathbb{E}[h(Z)]\} \\
& =\frac{(w-1) ! e^{\lambda}}{\lambda^{w}}\{\mathbb{E}[h(Z)]-\mathbb{E}[h(Z)]\}=0 .
\end{aligned}
$$

Assim, usando o Lema 1.2,

$$
f(w)+\sum_{l=w}^{\infty} \frac{(w-1) !}{l !} \lambda^{l-w}\{h(l)-\mathbb{E}[h(Z)]\}=0 .
$$


Lema 1.3 Para $h: \mathbb{Z}_{+} \rightarrow \mathbb{R}$ limitada, $f$ dada no Corolário 1.1 é limitada.

Prova: No caso de $w=0$ temos que $f(0)=0$. Por hipótese, existe $c>0$ tal que $|h(w)| \leq c$ para todo $w \in \mathbb{Z}_{+}$.

Para $w \geq 1$, temos que

$$
\begin{aligned}
|f(w)| & =\left|-\sum_{l=w}^{\infty} \frac{(w-1) !}{l !} \lambda^{l-w}\{h(l)-\mathbb{E}[h(Z)]\}\right| \\
& \leq \sum_{l=w}^{\infty} c\left|\frac{(w-1) !}{l !} \lambda^{l-w}\right|+c\left|\frac{(w-1) !}{l !} \lambda^{l-w}\right| \\
& =\sum_{l=w}^{\infty} 2 c\left|\frac{(w-1) !}{l !} \lambda^{l-w}\right| \\
& =2 c \sum_{l=w}^{\infty} \frac{(w-1) !}{l !} \lambda^{l-w} \\
& =2 c(w-1) !\left[\frac{1}{w !}+\frac{\lambda}{(w+1) !}+\cdots+\frac{\lambda^{j}}{(w+j) !}+\cdots\right] \\
& =2 c\left[\frac{1}{w}+\frac{\lambda}{(w+1) w}+\cdots+\frac{\lambda^{j}}{(w+j) \cdots w}+\cdots\right] \\
& \leq 2 c\left[\frac{1}{0 !}+\frac{\lambda}{1 !}+\cdots+\frac{\lambda^{j}}{j !}+\cdots\right] \\
& =2 c \sum_{k=0}^{\infty} \frac{\lambda^{k}}{k !}=2 c e^{\lambda},
\end{aligned}
$$

onde a última desigualdade segue do fato que $(w+n)(w+n-1) \cdots(w+1) w \geq n$ !, para $n \geq 0$.

O teorema a seguir é crucial no método de Chen-Stein. 
Teorema 1.1 Seja $W$ uma variável aleatória com valores em $\mathbb{Z}_{+}$. Então $\mathbb{E}[(T f)(W)]=0$ para toda $f: \mathbb{Z}_{+} \rightarrow \mathbb{R}$ limitada se, e somente se, $W \sim P(\lambda)$.

Prova: (Necessária) Seja $h: \mathbb{Z}_{+} \rightarrow \mathbb{R}$ limitada arbitrária e $f$ a solução da equação (1.1) (de Chen-Stein). Então

$$
\mathbb{E}[(T f)(W)]=\mathbb{E}[\lambda f(W+1)-W f(W)]=\mathbb{E}\{h(W)-\mathbb{E}[h(Z)]\}
$$

onde $Z \sim P(\lambda)$. Como $\mathbb{E}[(T f)(W)]=0$, temos que $\mathbb{E}\{h(W)-\mathbb{E}[h(Z)]\}=0$. Escolhendo $h(w)=\mathbf{1}_{A}(w)$ para $A \subset \mathbb{Z}_{+}$arbitrário, onde definimos $\mathbf{1}_{A}(w)$ a função indicadora em $\mathbb{Z}_{+}$por

$$
\mathbf{1}_{A}(w)= \begin{cases}1, & \text { se } w \in A \\ 0, & \text { se } w \notin A,\end{cases}
$$

temos que

$$
\mathbb{E}\left[\mathbf{1}_{A}(W)\right]-\mathbb{E}\left[\mathbf{1}_{A}(Z)\right]=\mathbb{P}(W \in A)-\mathbb{P}(Z \in A)=0 .
$$

Segue que $W \sim P(\lambda)$.

(Suficiente) Suponha $W \sim P(\lambda)$, então

$$
\begin{aligned}
\mathbb{E}[W f(W)] & =\sum_{w=0}^{\infty} w f(w) \frac{e^{-\lambda} \lambda^{w}}{w !}=\lambda \sum_{w=1}^{\infty} f(w) \frac{e^{-\lambda} \lambda^{w-1}}{(w-1) !} \\
& =\lambda \sum_{j=0}^{\infty} f(j+1) \frac{e^{-\lambda} \lambda^{j}}{j !}=\lambda \mathbb{E}[f(W+1)],
\end{aligned}
$$

isto é, $\lambda \mathbb{E}[f(W+1)]-\mathbb{E}[W f(W)]=0$ para toda $f: \mathbb{Z}_{+} \rightarrow \mathbb{R}$ limitada.

$\mathrm{O}$ método de Chen-Stein permite-nos obter cotas superiores na diferença entre $\mathbb{E}[(T f)(W)]$ e $\mathbb{E}[(T f)(Z)]$, onde $Z \sim P(\lambda)$. Em algumas situações as cotas superiores na distância em variação total podem ser dadas apenas em termos do primeiro e segundo momento da variável aleatória $W$.

O método proposto por Chen tem como base definir, de uma forma adequada "vizinhanças de dependência forte". Achá-las pode ser considerado uma "arte", já que elas determinam a aproximação. 
Seja $\Gamma$ um conjunto índice arbitrário, enumerável, e para cada $\alpha \in \Gamma$ considere $X_{\alpha}$ uma variável aleatória Bernoulli com $p_{\alpha}=\mathbb{P}\left(X_{\alpha}=1\right)=1-\mathbb{P}\left(X_{\alpha}=0\right)>0$. Defina $W$ por

$$
W=\sum_{\alpha \in \Gamma} X_{\alpha}, \quad \operatorname{com} \quad \mathbb{E}(W)=\lambda=\sum_{\alpha \in \Gamma} p_{\alpha} .
$$

Assumiremos que $\lambda \in(0, \infty)$.

Para cada $\alpha \in \Gamma$, definimos sua vizinhança de dependência como algum conjunto $B_{\alpha}$ tal que $\alpha \in B_{\alpha}$ e $B_{\alpha} \subset \Gamma$.

A distância em variação total da distribuição de probabilidade de $W$ e $Z$, onde $Z \sim P(\lambda)$, pode ser limitada superiormente em termos de três quantidades; $b_{1}, b_{2}, b_{3}\left(b_{3}^{\prime}\right)$, as quais são definidas por:

$$
b_{1}=\sum_{\alpha \in \Gamma} \sum_{\beta \in B_{\alpha}} \mathbb{E}\left(X_{\alpha}\right) \mathbb{E}\left(X_{\beta}\right)=\sum_{\alpha \in \Gamma} p_{\alpha} \sum_{\beta \in B_{\alpha}} p_{\beta},
$$

que permite avaliar a vizinhança de dependência,

$$
b_{2}=\sum_{\alpha \in \Gamma} \sum_{\substack{\beta \in B_{\alpha} \\ \beta \neq \alpha}} \mathbb{E}\left(X_{\alpha} X_{\beta}\right)=\sum_{\alpha \in \Gamma} \sum_{\substack{\beta \in B_{\alpha} \\ \beta \neq \alpha}} p_{\alpha \beta},
$$

que expressa as correlações entre os vizinhos de $B_{\alpha}$, e

$$
b_{3}=\sum_{\alpha \in \Gamma} s_{\alpha} \quad\left(\text { e } \quad b_{3}^{\prime}=\sum_{\alpha \in \Gamma} s_{\alpha}^{\prime}\right),
$$

onde

$$
\begin{aligned}
s_{\alpha} & =\mathbb{E}\left|\mathbb{E}\left(X_{\alpha}-p_{\alpha} / \sum_{\beta \in \Gamma-B_{\alpha}} X_{\beta}\right)\right| \\
& \leq s_{\alpha}^{\prime}=\mathbb{E}\left|\mathbb{E}\left[X_{\alpha}-p_{\alpha} / \sigma\left(X_{\beta}: \beta \in \Gamma-B_{\alpha}\right)\right]\right|,
\end{aligned}
$$

com $\sigma\left(X_{\beta}: \beta \in \Gamma-B_{\alpha}\right)$ denotando a $\sigma$-álgebra gerada pelo conjunto de variáveis aleatórias $X_{\beta}$, com $\beta \in \Gamma-B_{\alpha}$, que avalia a dependência entre um evento e o número de acontecimentos exteriores a sua vizinhança.

Para a última desigualdade tomemos o seguinte resultado (ver [8] página 196, Teorema 1.2); se $\mathcal{F}_{1} \subset \mathcal{F}_{2} \sigma$-álgebras, e $X$ uma variável aleatória então

$$
\mathbb{E}\left[\mathbb{E}\left(X / \mathcal{F}_{2}\right) / \mathcal{F}_{1}\right]=\mathbb{E}\left(X / \mathcal{F}_{1}\right) \quad \text { quase-certamente }
$$




$$
\begin{aligned}
& \text { Como } \beta \in \Gamma-B_{\alpha}=B_{\alpha}^{c} \text { e } \sum_{\beta \in B_{\alpha}^{c}} X_{\beta} \subset \sigma\left(X_{\beta}: \beta \in \Gamma-B_{\alpha}\right) \text { então } \\
& \mathbb{E}\left|\mathbb{E}\left(X_{\alpha}-p_{\alpha} / \sum_{\beta \in B_{\alpha}^{c}} X_{\beta}\right)\right|=\mathbb{E}\left|\mathbb{E}\left\{\mathbb{E}\left[X_{\alpha}-p_{\alpha} / \sigma\left(X_{\beta}: \beta \in B_{\alpha}^{c}\right)\right] / \sum_{\beta \in B_{\alpha}^{c}} X_{\beta}\right\}\right| \\
& \leq \mathbb{E}\left\{\mathbb{E}\left[\left|\mathbb{E}\left(X_{\alpha}-p_{\alpha} / \sigma\left(X_{\beta}: \beta \in B_{\alpha}^{c}\right)\right)\right| / \sum_{\beta \in B_{\alpha}^{c}} X_{\beta}\right]\right\} \\
&=\mathbb{E}\left|\mathbb{E}\left[X_{\alpha}-p_{\alpha} / \sigma\left(X_{\beta}: \beta \in B_{\alpha}^{c}\right)\right]\right|
\end{aligned}
$$

o qual prova que $s_{\alpha} \leq s_{\alpha}^{\prime}$ e portanto $b_{3} \leq b_{3}^{\prime}$.

$\mathrm{O}$ cálculo de $b_{1}$ e $b_{2}$, é equivalente a achar o primeiro e segundo momentos de $W$. Os dois casos envolvem as quantidades $p_{\alpha}$ e $p_{\alpha \beta}$. Quando for possivel tomar uma vizinhança tal que $X_{\alpha}$ e $X_{\beta}$ são independentes $\operatorname{com} \beta \in \Gamma-B_{\alpha}$, de modo que $b_{3}=0\left(b_{3}^{\prime}=0\right)$, temos que a diferença entre $b_{2}$ e $b_{1}$ pode ser escrita como $b_{2}-b_{1}=\mathbb{E}\left(W^{2}\right)-\lambda-\lambda^{2}=\mathbb{E}\left(W^{2}\right)-\mathbb{E}\left(Z^{2}\right)$.

De fato, para $W=\sum_{\alpha \in \Gamma} X_{\alpha}, \quad \lambda=\sum_{\alpha \in \Gamma} p_{\alpha}$ e $Z \sim P(\lambda)$, temos que

$$
\begin{aligned}
\mathbb{E}\left(W^{2}\right)-\mathbb{E}\left(Z^{2}\right)= & \mathbb{E}\left(\sum_{\alpha \in \Gamma} X_{\alpha}\right)^{2}-\mathbb{E}\left(Z^{2}\right) \\
= & \sum_{\alpha \in \Gamma} \mathbb{E}\left(X_{\alpha}^{2}\right)+\sum_{\alpha \in \Gamma} \sum_{\beta \neq \alpha} \mathbb{E}\left(X_{\alpha} X_{\beta}\right)-\lambda-\lambda^{2} \\
= & \sum_{\alpha \in \Gamma}\left[p_{\alpha}\left(1-p_{\alpha}\right)+p_{\alpha}^{2}\right]+ \\
& \sum_{\alpha \in \Gamma}\left[\sum_{\substack{\beta \in B_{\alpha} \\
\beta \neq \alpha}} \mathbb{E}\left(X_{\alpha} X_{\beta}\right)+\sum_{\beta \notin B_{\alpha}} \mathbb{E}\left(X_{\alpha}\right) \mathbb{E}\left(X_{\beta}\right)\right]-\lambda-\lambda^{2} \\
= & \sum_{\alpha \in \Gamma} p_{\alpha}+\sum_{\substack { \alpha \in \Gamma \\
\begin{subarray}{c}{\beta \in B_{\alpha} \\
\beta \neq \alpha{ \alpha \in \Gamma \\
\begin{subarray} { c } { \beta \in B _ { \alpha } \\
\beta \neq \alpha } }\end{subarray}} \mathbb{E}\left(X_{\alpha} X_{\beta}\right)+\sum_{\alpha \in \Gamma} \sum_{\substack{\beta \in \Gamma \\
\beta \neq \alpha}} \mathbb{E}\left(X_{\alpha}\right) \mathbb{E}\left(X_{\beta}\right)- \\
& \sum_{\alpha \in \Gamma} \sum_{\substack{\beta \in B_{\alpha} \\
\beta \neq \alpha}} \mathbb{E}\left(X_{\alpha}\right) \mathbb{E}\left(X_{\beta}\right)-\lambda-\lambda^{2} \\
= & b_{2}+\lambda^{2}-p_{\alpha \alpha}-b_{1}+p_{\alpha \alpha}-\lambda^{2} \\
= & b_{2}-b_{1} .
\end{aligned}
$$

Em algumas situações, as probabilidades $p_{\alpha}$ e o cardinal da vizinhança de $\alpha$ 
são constantes para $\alpha \in \Gamma$. Neste caso, $b_{1}$ pode ser escrito como $b_{1}=|\Gamma|\left|B_{\alpha}\right| p_{\alpha}^{2} \mathrm{e}$ $\lambda^{2}=\left(|\Gamma| p_{\alpha}\right)^{2}$. Portanto $b_{1}=\frac{\lambda^{2}\left|B_{\alpha}\right|}{|\Gamma|}$. Para $\lambda$ fixado, $b_{1}$ é pequeno se a vizinhança de $\alpha$ é pequena comparada ao conjunto de índices.

O principal resultado deste capítulo é o Teorema 1.2. Esse teorema apresenta um limite superior da distância em variação total dada pelas quantidades $b_{1}, b_{2}$ e $b_{3}\left(b_{3}^{\prime}\right)$ que foram definidas anteriormente.

Teorema 1.2 Seja $\Gamma$ um conjunto índice arbitrário enumerável e para cada $\alpha \in$ $\Gamma$, seja $X_{\alpha}$ uma variável aleatória Bernoulli com probabilidade $p_{\alpha}=\mathbb{P}\left(X_{\alpha}=\right.$ $1)=1-\mathbb{P}\left(X_{\alpha}=0\right)>0$. Além disso, sejam $W=\sum_{\alpha \in \Gamma} X_{\alpha}$ e $Z \sim P(\lambda)$ com $\mathbb{E}(Z)=\mathbb{E}(W)=\lambda<\infty$. Então

$$
\begin{aligned}
d_{V T}(W, Z) & \leq\left(b_{1}+b_{2}\right) \frac{1-e^{-\lambda}}{\lambda}+b_{3} \min \left(1, c_{1} \lambda^{-\frac{1}{2}}\right) \\
& \leq b_{1}+b_{2}+b_{3}^{\prime}
\end{aligned}
$$

para algum $c_{1}>0$. Além disso

$$
\begin{aligned}
\left|\mathbb{P}(W=0)-e^{-\lambda}\right| & \leq\left(b_{1}+b_{2}+b_{3}\right) \frac{1-e^{-\lambda}}{\lambda} \\
& <\min \left(1, \lambda^{-1}\right)\left(b_{1}+b_{2}+b_{3}^{\prime}\right)
\end{aligned}
$$

onde $\mathbb{P}$ é a lei da variável aleatória $W$.

Para provar o Teorema 1.2, precisamos de alguns resultados, os quais apresentaremos nos lemas a seguir.

Lema $1.4 Z \sim P(\lambda)$ satisfaz a recursão

$$
\lambda \mathbb{P}(Z=k-1)=k \mathbb{P}(Z=k), \quad k=0,1,2, \ldots .
$$

Prova: Se $k=0$, temos

$$
\lambda \mathbb{P}(Z=-1)=0 \mathbb{P}(Z=0) .
$$

Se $k>0$, temos

$$
\lambda \mathbb{P}(Z=k-1)=\lambda \frac{e^{-\lambda} \lambda^{k-1}}{(k-1) !}=k \frac{e^{-\lambda} \lambda^{k}}{k !}=k \mathbb{P}(Z=k) .
$$


Lema 1.5 Para $w \geq 1$ e $\lambda \geq w$ temos que

$$
(w-1) ! \lambda^{-w} \sum_{l=0}^{w-1} \frac{\lambda^{l}}{l !} \leq 2 \lambda^{-\frac{1}{2}} .
$$

Prova: Para $w=1$, então $\lambda \geq \frac{1}{4}$ de onde $\sqrt{\lambda} \geq \frac{1}{2}$ implica que $2 \geq \frac{1}{\sqrt{\lambda}}$ e portanto $\frac{1}{\lambda} \leq \frac{2}{\sqrt{\lambda}}$.

Para $w \geq 2$, tomemos $\phi(\lambda): \mathbb{R}_{+} \rightarrow \mathbb{R}$ uma função crescente de $\lambda$ tal que $1 \leq \phi(\lambda)<\lambda$ e $\phi(\lambda) \rightarrow \infty$, quando $\lambda \rightarrow \infty$. Então

$$
\begin{aligned}
& (w-1) ! \lambda^{-w} \sum_{l=0}^{w-1} \frac{\lambda^{l}}{l !}=(w-1) ! \lambda^{-w}\left[1+\frac{\lambda}{1 !}+\frac{\lambda^{2}}{2 !}+\cdots+\frac{\lambda^{w-2}}{(w-2) !}+\frac{\lambda^{w-1}}{(w-1) !}\right] \\
& =\frac{1}{\lambda}+\frac{(w-1)}{\lambda^{2}}+\cdots+\frac{(w-1)(w-2) \cdots 3}{\lambda^{w-2}}+\frac{(w-1) !}{\lambda^{w-1}}+\frac{(w-1) !}{\lambda^{w}} \\
& =\frac{1}{\lambda}+\sum_{l=1}^{w-1} \frac{(w-1)(w-2) \cdots(w-l)}{\lambda^{l+1}} .
\end{aligned}
$$

Denotando o maior inteiro menor ou igual a $r \in \mathbb{R}$ por $|r|$, temos que

$$
\begin{aligned}
(w & -1) ! \lambda^{-w} \sum_{l=0}^{w-1} \frac{\lambda^{l}}{l !} \leq \frac{1}{\lambda}+\sum_{l=1}^{|\lambda|-1} \frac{(\lambda-1)(\lambda-2) \cdots(\lambda-l)}{\lambda^{l+1}} \\
& =\frac{1}{\lambda}+\sum_{l=1}^{|\phi(\lambda)|-1} \frac{(\lambda-1)(\lambda-2) \cdots(\lambda-l)}{\lambda^{l+1}}+\sum_{l=|\phi(\lambda)|}^{|\lambda|-1} \frac{(\lambda-1)(\lambda-2) \cdots(\lambda-l)}{\lambda^{l+1}} \\
& \leq \frac{1}{\lambda}+\sum_{l=1}^{|\phi(\lambda)|-1} \frac{\lambda^{l}}{\lambda^{l+1}}+\sum_{l=\lfloor\phi(\lambda) \mid} \frac{|\lambda|-1}{\lambda^{l+1}} \\
& =\frac{1}{\lambda}+\frac{1}{\lambda}\{|\phi(\lambda)|-1\}+\sum_{l=|\phi(\lambda)|}^{|\lambda|-1} \frac{(\lambda-1)(\lambda-2) \cdots(\lambda-l)}{\lambda^{l+1}} \\
& =\frac{|\phi(\lambda)|}{\lambda}+\sum_{l=|\phi(\lambda)|}^{|\lambda|-1} \frac{(\lambda-1)(\lambda-2) \cdots(\lambda-l)}{\lambda^{l+1}} \\
& \leq \frac{\phi(\lambda)}{\lambda}+\sum_{l=|\phi(\lambda)|}^{|\lambda|-1} \frac{(\lambda-1)(\lambda-2) \cdots(\lambda-l)}{\lambda^{l+1}} .
\end{aligned}
$$


Como

$$
\begin{aligned}
& \sum_{l=\lfloor\phi(\lambda) \mid}^{|\lambda|-1} \frac{(\lambda-1)(\lambda-2) \cdots(\lambda-l)}{\lambda^{l+1}} \\
& =\frac{(\lambda-1)(\lambda-2) \cdots(\lambda-\mid \phi(\lambda) \|+1)(\lambda-\mid \phi(\lambda) \|)}{\lambda^{\|\phi(\lambda)\|+1}} \\
& +\frac{(\lambda-1)(\lambda-2) \cdots(\lambda-\llbracket \phi(\lambda) \rrbracket+1)(\lambda-\llbracket \phi(\lambda) \rrbracket)(\lambda-\llbracket \phi(\lambda) \rrbracket-1)}{\lambda \llbracket \phi(\lambda) \rrbracket+2}+ \\
& \cdots+\frac{(\lambda-1)(\lambda-2) \cdots(\lambda-\mid \phi(\lambda) \rrbracket) \cdots(\lambda-\| \lambda \rrbracket+2)(\lambda-\llbracket \lambda \|+1)}{\lambda \llbracket \lambda \rrbracket} \\
& =\frac{(\lambda-1)(\lambda-2) \cdots(\lambda-\llbracket \phi(\lambda) \rrbracket+1)(\lambda-\llbracket \phi(\lambda) \rrbracket)}{\lambda_{\lambda} \mid \phi(\lambda) \rrbracket}\left[\frac{1}{\lambda}+\frac{\lambda-\llbracket \phi(\lambda) \rrbracket-1}{\lambda^{2}}+\right. \\
& \left.\cdots+\frac{(\lambda-|\phi(\lambda)|-1) \cdots(\lambda-\| \lambda \rrbracket+2)(\lambda-\llbracket \lambda \mid+1)}{\lambda \mid \lambda \rrbracket-\llbracket \phi(\lambda) \rrbracket}\right] \\
& \frac{(\lambda-1)(\lambda-2) \cdots(\lambda-\llbracket \phi(\lambda) \rrbracket+1)(\lambda-\| \phi(\lambda) \rrbracket)}{\lambda^{\| \phi(\lambda) \rrbracket}} \leq \frac{\lambda^{\mid \phi(\lambda) \rrbracket}}{\lambda^{\| \phi(\lambda) \rrbracket}}=1 .
\end{aligned}
$$

Observando que $\lambda-(|\phi(\lambda)|+1) \leq \lambda-\phi(\lambda)$ temps que

$$
\begin{gathered}
\sum_{l=|\phi(\lambda)|}^{|\lambda|-1} \frac{(\lambda-1)(\lambda-2) \cdots(\lambda-l)}{\lambda^{l+1}} \leq \frac{1}{\lambda}+\frac{\lambda-(|\phi(\lambda)|+1)}{\lambda^{2}}+ \\
\cdots+\frac{[\lambda-(|\phi(\lambda)|+1)] \cdots[\lambda-(|\lambda|-2)][\lambda-(|\lambda|-1)]}{\lambda|\lambda|-|\phi(\lambda)|} \\
\leq \quad \frac{1}{\lambda}+\frac{\lambda-(|\phi(\lambda)|+1)}{\lambda^{2}}+ \\
\quad \cdots+\frac{[\lambda-(|\phi(\lambda)|+1)] \cdots[\lambda-(|\phi(\lambda)|-2)][\lambda-(|\phi(\lambda)|-1)]}{\lambda|\lambda|-|\phi(\lambda)|} \\
\leq \frac{1}{\lambda}+\frac{(\lambda-\phi(\lambda))}{\lambda^{2}}+\cdots+\frac{(\lambda-\phi(\lambda))|\lambda|-|\phi(\lambda)|-1}{\lambda|\lambda|-|\phi(\lambda)|}
\end{gathered}
$$

13 


$$
=\sum_{l=0}^{\|\lambda\|-\llbracket \phi(\lambda) \|^{-1}} \frac{(\lambda-\phi(\lambda))^{l}}{\lambda^{l+1}} \leq \sum_{l=0}^{\infty} \frac{(\lambda-\phi(\lambda))^{l}}{\lambda^{l+1}}
$$

e portanto

$$
\begin{aligned}
(w-1) ! \lambda^{-w} \sum_{l=0}^{w-1} \frac{\lambda^{l}}{l !} & \leq \frac{\phi(\lambda)}{\lambda}+\sum_{l=0}^{\infty} \frac{(\lambda-\phi(\lambda))^{l}}{\lambda^{l+1}}=\frac{\phi(\lambda)}{\lambda}+\frac{1}{\lambda} \sum_{l=0}^{\infty}\left(\frac{\lambda-\phi(\lambda)}{\lambda}\right)^{l} \\
& =\frac{\phi(\lambda)}{\lambda}+\frac{1}{\lambda} \frac{1}{1-\frac{(\lambda-\phi(\lambda))}{\lambda}}=\frac{\phi(\lambda)}{\lambda}+\frac{1}{\lambda} \frac{\lambda}{\phi(\lambda)} \\
& =\frac{\phi(\lambda)}{\lambda}+\frac{1}{\phi(\lambda)} .
\end{aligned}
$$

Para finalizar basta escolher $\phi(\lambda)=\lambda^{\frac{1}{2}}$, e temos o resultado, isto é,

$$
(w-1) ! \lambda^{-w} \sum_{l=0}^{w-1} \frac{\lambda^{l}}{l !} \leq \frac{\lambda^{\frac{1}{2}}}{\lambda}+\frac{1}{\lambda^{\frac{1}{2}}}=2 \lambda^{-\frac{1}{2}} .
$$

Lema 1.6 Para $w \geq 1$ e $0<\lambda \leq w$ temos que

$$
(w-1) ! \lambda^{-w} \sum_{l=w}^{\infty} \frac{\lambda^{l}}{l !} \leq 2 w^{-\frac{1}{2}} .
$$

Prova: Dividiremos a prova em quatro casos.

Caso $w=1$; provemos que $\frac{1}{\lambda} \sum_{l=1}^{\infty} \frac{\lambda^{l}}{l !} \leq 2$. Como $\sum_{l=1}^{\infty} \frac{\lambda^{l}}{l !}=e^{\lambda}-1$ então basta verificar que $e^{\lambda}-1-2 \lambda \leq 0$ para todo $\lambda \in(0,1]$.

Seja $g(\lambda)=e^{\lambda}-1-2 \lambda$. Temos que $g^{(1)}(\lambda)=e^{\lambda}-2$, onde $g^{(1)}$ denota a derivada da função $g$ em relação a $\lambda$. Em geral para qualquer função $f$, vamos definir $f^{(n)}$ como a $n$-ésima derivada de $f$ em relação a $\lambda$. Assim, $g^{(1)}(\lambda)=0$ somente se $\lambda=\ln (2)$. Além disso,

$$
\begin{aligned}
& g^{(1)}(\lambda)<0 \quad \text { para } 0<\lambda<\ln (2) \\
& g^{(1)}(\lambda)>0 \quad \text { para } \ln (2)<\lambda \leq 1
\end{aligned}
$$


de onde concluímos que $g(\lambda)$ é decrescente no intervalo $(0, \ln (2)]$ e crescente no intervalo $(\ln (2), 1]$ e como $g(0)=0, g(1)<0$ temos que $g(\lambda)<0$ para todo $\lambda \in(0,1]$.

Caso $w=2$; o lema afirma que $\frac{1}{\lambda^{2}} \sum_{l=2}^{\infty} \frac{\lambda^{l}}{l !} \leq \frac{2}{\sqrt{2}}=\sqrt{2}$. Tomemos a função $q(\lambda)=e^{\lambda}-1-\lambda-\sqrt{2} \lambda^{2}$ e vejamos que $q(\lambda) \leq 0$ para todo $\lambda \in(0,2]$.

Como $q^{(1)}(\lambda)=e^{\lambda}-1-2 \sqrt{2} \lambda, q^{(2)}(\lambda)=e^{\lambda}-2 \sqrt{2}$, de onde $q^{(2)}(\lambda)=0$ somente se $\lambda=\ln (2 \sqrt{2})$. Além disso,

$$
\begin{aligned}
& q^{(2)}(\lambda)<0 \quad \text { para } 0<\lambda<\ln (2 \sqrt{2}) \\
& q^{(2)}(\lambda)>0 \text { para } \ln (2 \sqrt{2})<\lambda \leq 2
\end{aligned}
$$

portanto $q^{(1)}(\lambda)$ é decrescente no intervalo $(0, \ln (2 \sqrt{2})]$ e crescente no intervalo $(\ln (2 \sqrt{2}), 2]$. Como $q^{(1)}(0)=0$ e $q^{(1)}(2)>0$, então existe um único $\lambda^{*} \in(0,2]$ tal que $q^{(1)}\left(\lambda^{*}\right)=0$ de onde concluímos que $q(\lambda)$ é decrescente no intervalo $\left(0, \lambda^{*}\right]$ e crescente em $\left(\lambda^{*}, 2\right]$. Além disso, $q(0)=0$ e $q(2)<0$, concluímos que $q(\lambda) \leq 0$ para todo $\lambda \in(0,2]$.

Caso $w=3$; Temos $\frac{2}{\lambda^{3}} \sum_{l=3}^{\infty} \frac{\lambda^{l}}{l !} \leq \frac{2}{\sqrt{3}}$, isto é $\frac{2}{\lambda^{3}}\left(e^{\lambda}-1-\lambda-\frac{\lambda^{2}}{2}\right) \leq \frac{2}{\sqrt{3}}$ ou seja $e^{\lambda}-1-\lambda-\frac{\lambda^{2}}{2}-\frac{\lambda^{3}}{\sqrt{3}} \leq 0$. Tomemos agora a função $r(\lambda)=e^{\lambda}-1-\lambda-\frac{\lambda^{2}}{2}-\frac{\lambda^{3}}{\sqrt{3}}$. Precisamos mostrar que $r(\lambda) \leq 0$ para todo $\lambda \in(0,3]$. Temos que $r^{(4)}(\lambda)=e^{\lambda}>0$ para todo $\lambda \in(0,3]$. Daqui $r^{(3)}(\lambda)=e^{\lambda}-\frac{6}{\sqrt{3}}$ é crescente no intervalo $(0,3] \mathrm{e}$ $r^{(3)}(0)<0, r^{(3)}(3)>0$, portanto existe um único $\lambda_{1} \in(0,3]$ tal que $r^{(3)}(\lambda) \leq 0$ para $\lambda \in\left(0, \lambda_{1}\right]$ e $r^{(3)}(\lambda)>0$ para $\lambda \in\left(\lambda_{1}, 3\right]$. De novo, segue que $r^{(2)}(\lambda)=$ $e^{\lambda}-1-\frac{6 \lambda}{\sqrt{3}}$ é decrescente em $\left(0, \lambda_{1}\right]$ e crescente em $\left(\lambda_{1}, 3\right]$, com $r^{(2)}(0)<0$ e $r^{(2)}(3) \geq 0$, logo existe um único $\lambda_{2} \in(0,3]$ tal que $r^{(2)}(\lambda) \leq 0$ no $\left(0, \lambda_{2}\right]$ e $r^{(2)}(\lambda)>0$ no intervalo $\left(\lambda_{2}, 3\right]$. Da mesma maneira, $r^{(1)}(\lambda)=e^{\lambda}-1-\lambda-\frac{3 \lambda^{2}}{\sqrt{3}}$ é decrescente para $\lambda \in\left(0, \lambda_{2}\right]$ e é crescente para $\lambda \in\left(\lambda_{2}, 3\right]$. Como $r^{(1)}(0)=0$ e $r^{(1)}(3)>0$ então existe um único $\lambda_{3} \in(0,3]$ tal que $r^{(1)}(\lambda) \leq 0 \operatorname{com} \lambda \in\left(0, \lambda_{3}\right]$ e $r^{(1)}(\lambda)>0$ para $\lambda \in\left(\lambda_{3}, 3\right]$. Finalmente $r(\lambda)$ é decrescente com $\lambda \in\left(0, \lambda_{3}\right]$ e crescente em $\left(\lambda_{3}, 3\right]$, com $r(0)=0$ e $r(3)<0$, segue que $r(\lambda) \leq 0$ para todo $\lambda \in(0,3]$.

Caso $w \geq 4$; temos que 


$$
\begin{aligned}
(w-1) ! \lambda^{-w^{\prime}} & \sum_{l=w}^{\infty} \frac{\lambda^{l}}{l !}=(w-1) ! \lambda^{-w}\left[\frac{\lambda^{w}}{w !}+\frac{\lambda^{w+1}}{(w+1) !}+\cdots+\frac{\lambda^{w+j}}{(w+j) !}+\cdots\right] \\
& =\frac{1}{w}+\frac{\lambda}{w(w+1)}+\cdots+\frac{\lambda^{j}}{w(w+1) \cdots(w+j)}+\cdots \\
& \leq \frac{1}{w}+\sum_{l=1}^{\infty} \frac{w^{l}}{w(w+1) \cdots(w+l)} \\
& =\frac{1}{w}+\sum_{l=1}^{\mid w^{\frac{1}{2}} \rrbracket-2} \frac{w^{l}}{w(w+1) \cdots(w+l)}+\sum_{l=\left\lfloor w^{\frac{1}{2}} \rrbracket-1\right.}^{\infty} \frac{w^{l}}{w(w+1) \cdots(w+l)} .
\end{aligned}
$$

Agora. observe que:

i)

$$
\begin{aligned}
\frac{1}{w}+\sum_{l=1}^{\left|w^{\frac{1}{2}}\right|-2} \frac{w^{l}}{w(w+1) \cdots(w+l)} & \leq \frac{1}{w}+\sum_{l=1}^{\left|w^{\frac{1}{2}}\right|-2} \frac{w^{l}}{w^{l+1}}=\frac{1}{w}+\frac{1}{w} \sum_{l=1}^{\llbracket w^{\frac{1}{2}} \mid-2} \frac{w^{l}}{w^{l}} \\
& =\frac{1}{w}+\frac{1}{w}\left(\| w^{\frac{1}{2}} \rrbracket-2\right)=\frac{1}{w}\left(\| w^{\frac{1}{2}} \rrbracket-1\right) .
\end{aligned}
$$

ii)

$$
\begin{aligned}
& \sum_{l=\left|w^{\frac{1}{2}}\right|-1}^{\infty} \frac{w^{l-1}}{(w+1) \cdots(w+l)}=\frac{w^{\left|w^{\frac{1}{2}}\right|-2}}{(w+1) \cdots\left(w+\left|w^{\frac{1}{2}}\right|-1\right)} \\
& +\frac{w^{\left|w^{\frac{1}{2}}\right|-1}}{(w+1) \cdots\left(w+\left|w^{\frac{1}{2}}\right|-1\right)\left(w+\left|w^{\frac{1}{2}}\right|\right)} \\
& +\frac{w^{\left|w^{\frac{1}{2}}\right|}}{(w+1) \cdots\left(w+\left|w^{\frac{1}{2}}\right|\right)\left(w+\left|w^{\frac{1}{2}}\right|+1\right)}+\cdots \\
& +\frac{w^{j-1}}{(w+1) \cdots\left(w+\left|w^{\frac{1}{2}}\right|\right) \cdots(w+j)}+\cdots
\end{aligned}
$$




$$
\begin{aligned}
& =\frac{w^{\llbracket w^{\frac{1}{2}} \mid-1}}{(w+1) \cdots\left(w+\left|w^{\frac{1}{2}}\right|-1\right)} \times \\
& {\left[\frac{1}{w}+\frac{1}{\left(w+\left|w^{\frac{1}{2}}\right|\right)}+\frac{w}{\left(w+\left|w^{\frac{1}{2}}\right|\right)\left(w+\left|w^{\frac{1}{2}}\right|+1\right)}+\right.} \\
& \left.\cdots+\frac{w^{j-\left\lfloor w^{\frac{1}{2}}\right\rceil}}{\left(w+\left\lfloor w^{\frac{1}{2}} \mid\right)\left(w+\llbracket w^{\frac{1}{2}} \rrbracket+1\right) \cdots(w+j)\right.}+\cdots\right] \\
& \leq \frac{1}{w}+\frac{1}{w+\left|w^{\frac{1}{2}}\right|} \times \\
& {\left[1+\frac{w}{\left(w+\left|w^{\frac{1}{2}}\right|+1\right)}+\cdots+\frac{w^{j-\left|w^{\frac{1}{2}}\right|}}{\left(w+\left|w^{\frac{1}{2}}\right|+1\right) \cdots(w+j)}+\cdots\right],} \\
& \sum_{l=\left|w^{\frac{1}{2}}\right|-1}^{\infty} \frac{w^{l-1}}{(w+1) \cdots(w+l)} \\
& \leq \frac{1}{w}+\frac{1}{w+\left|w^{\frac{1}{2}}\right|}\left[1+\frac{w}{\left(w+w^{\frac{1}{2}}\right)}+\cdots+\frac{w^{j-\left|w^{\frac{1}{2}}\right|}}{\left(w+w^{\frac{1}{2}}\right)^{j-\left|w^{\frac{1}{2}}\right|}}+\cdots\right] \\
& =\frac{1}{w}+\frac{1}{w+\left\lfloor w^{\frac{1}{2}}\right\rceil} \sum_{l=0}^{\infty}\left(\frac{w}{w+w^{\frac{1}{2}}}\right)^{l} \text {. }
\end{aligned}
$$

Com (i), (ii) e usando o fato que $w^{\frac{1}{2}} \leq w+\left|w^{\frac{1}{2}}\right|$ temos que

$$
\begin{aligned}
(w-1) \lambda^{-w} \sum_{l=w}^{\infty} \frac{\lambda^{l}}{l !} & \leq \frac{\left|w^{\frac{1}{2}}\right|}{w}-\frac{1}{w}+\frac{1}{w}+\frac{1}{w+\left|w^{\frac{1}{2}}\right|} \sum_{l=0}^{\infty}\left(\frac{w}{w+w^{\frac{1}{2}}}\right)^{l} \\
& =\frac{1}{w}\left\{\left|w^{\frac{1}{2}}\right|+\frac{w}{w+\left|w^{\frac{1}{2}}\right|} \frac{w+w^{\frac{1}{2}}}{w^{\frac{1}{2}}}\right\} \\
& =\frac{1}{w^{\frac{1}{2}}}\left\{1+\frac{\left|w^{\frac{1}{2}}\right|}{w^{\frac{1}{2}}}+\frac{w\left(w+w^{\frac{1}{2}}\right)}{w\left(w+\left|w^{\frac{1}{2}}\right|\right)}-1\right\} \\
& =\frac{1}{w^{\frac{1}{2}}}\left\{1+\frac{\left|w^{\frac{1}{2}}\right|}{w^{\frac{1}{2}}}+\frac{w^{\frac{1}{2}}-\left|w^{\frac{1}{2}}\right|}{w+\left|w^{\frac{1}{2}}\right|}\right\}
\end{aligned}
$$

17 


$$
\begin{aligned}
& \leq \frac{1}{w^{\frac{1}{2}}}\left\{1+\frac{\llbracket w^{\frac{1}{2}} \rrbracket}{w^{\frac{1}{2}}}+\frac{w^{\frac{1}{2}}-\llbracket w^{\frac{1}{2}} \rrbracket}{w^{\frac{1}{2}}}\right\} \\
& =\frac{1}{w^{\frac{1}{2}}}\left\{1+\frac{\llbracket w^{\frac{1}{2}} \rrbracket}{w^{\frac{1}{2}}}+1-\frac{\llbracket w^{\frac{1}{2}} \rrbracket}{w^{\frac{1}{2}}}\right\} \\
& =\frac{1}{w^{\frac{1}{2}}}[2]=2 w^{-\frac{1}{2}} .
\end{aligned}
$$

Pelo Lema 1.3 temos que para $h: \mathbb{Z}_{+} \rightarrow \mathbb{R}$ limitada, a solução $f$ da equação (1.1) (de Chen-Stein) satisfaz $|f(w)| \leq 2 c e^{\lambda}, w \geq 1$ e $c>0$, mas isto pode ser refinado, como veremos no Lema 1.7 .

Definição 1.2 Para $f: \mathbb{Z}_{+} \rightarrow \mathbb{R}$ definimos a norma do supremo por

$$
\|f\|=\sup _{w \geq 0}|f(w)| .
$$

Lema 1.7 Para $h: \mathbb{Z}_{+} \rightarrow \mathbb{R}$ limitada a solução $f: \mathbb{Z}_{+} \rightarrow \mathbb{R}$ da equação (1.1) (de Chen-Stein) satisfaz

$$
\|f\| \leq 4\|h\| \min \left(\lambda^{-\frac{1}{2}}, 1\right) .
$$

Prova: Dividiremos a prova em dois casos.

Caso $\lambda \geq w \geq 1$. Aplicando os Lemas 1.2 e 1.5 temos

$$
\begin{aligned}
|f(w)| & =\left|(w-1) ! \lambda^{-w} \sum_{l=0}^{w-1} \frac{\lambda^{l}}{l !}\{h(l)-\mathbb{E}[h(Z)]\}\right| \\
& \leq(w-1) ! \lambda^{-w} \sum_{l=0}^{w-1}\left|\frac{\lambda^{l}}{l !} h(l)-\frac{\lambda^{l}}{l !} \mathbb{E}[h(Z)]\right| \\
& \leq(w-1) ! \lambda^{-w} \sum_{l=0}^{w-1}\left\{\left|\frac{\lambda^{l}}{l !} h(l)\right|+\left|\frac{\lambda^{l}}{l !} \mathbb{E}[h(Z)]\right|\right\} \\
& \leq(w-1) ! \lambda^{-w}\left\{\|h\| \sum_{l=0}^{w-1} \frac{\lambda^{l}}{l !}+\|h\| \sum_{l=0}^{w-1} \frac{\lambda^{l}}{l !}\right\} \\
& =2\|h\|(w-1) ! \lambda^{-w} \sum_{l=0}^{w-1} \frac{\lambda^{l}}{l !} \\
& \leq 4\|h\| \lambda^{-\frac{1}{2}} .
\end{aligned}
$$


Caso $w \geq 1$ e $0<\lambda \leq w$. Aplicando o Corolário 1.1 e o Lema 1.6

$$
\begin{aligned}
|f(w)| & =\left|-(w-1) ! \lambda^{-w} \sum_{l=w}^{\infty} \frac{\lambda^{l}}{l !}\{h(l)-\mathbb{E}[h(Z)]\}\right| \\
& \leq 2\|h\|(w-1) ! \lambda^{-w} \sum_{l=w}^{\infty} \frac{\lambda^{l}}{l !} \\
& \leq 4\|h\| w^{-\frac{1}{2}} .
\end{aligned}
$$

A condição $w \geq 1$ implica em $w^{-\frac{1}{2}} \leq 1$ e portanto $\min \left(\lambda^{-\frac{1}{2}}, w^{-\frac{1}{2}}\right) \leq \min \left(\lambda^{-\frac{1}{2}}, 1\right)$. Segue que

$$
\|f\| \leq 4\|h\| \min \left(\lambda^{-\frac{1}{2}}, w^{-\frac{1}{2}}\right) \leq 4\|h\| \min \left(\lambda^{-\frac{1}{2}}, 1\right) .
$$

Para $Z \sim P(\lambda)$, a equação de Chen-Stein dada em termos do operador linear $T$ com dependência do parâmetro $\lambda$, isto é,

$$
\lambda f(w+1)-w f(w)=(T f)(w)=h(w)-\mathbb{E}[h(Z)], \quad \text { para } w \geq 0,
$$

tem como solução $f(w)$ dada pela equação (1.2) que para $h: \mathbb{Z}_{+} \rightarrow \mathbb{R}$ limitada, pode ser escrita para $w \geq 1$ por

$$
\begin{aligned}
f(w) & =\frac{(w-1) ! \lambda^{-w} \lambda}{\lambda e^{-\lambda}} \sum_{l=0}^{w-1} \frac{e^{-\lambda} \lambda^{l}}{l !}(T f)(l) \\
& =\frac{1}{\lambda} \frac{(w-1) ! \lambda^{-w+1}}{e^{-\lambda}} \sum_{l=0}^{w-1} \frac{e^{-\lambda} \lambda^{l}}{l !}(T f)(l) \\
& =\frac{1}{\lambda \mathbb{P}(Z=w-1)} \sum_{l=0}^{w-1}(T f)(l) \mathbb{P}(Z=l) \\
& =\frac{\mathbb{E}\left[(T f)(Z) \mathbf{1}_{\{0,1,2, \ldots, w-1\}}(Z)\right]}{\lambda \mathbb{P}(Z=w-1)} \\
& =\frac{\mathbb{E}\left\{[h(Z)-\mathbb{E}[h(Z)]] \mathbf{1}_{\{0,1, \cdots, w-1\}}(Z)\right\}}{\lambda \mathbb{P}(Z=w-1)} .
\end{aligned}
$$

No caso em que $\mathbb{E}[h(Z)]=0$, representaremos a forma resultante pelo operador $S$, definido por

$$
(S h)(w)= \begin{cases}\frac{\mathbb{E}\left[h(Z) 1_{\{0,1, \cdots, w-1\}}(Z)\right]}{\lambda \mathbb{P}(Z=w-1)}, & \text { para } w \geq 1 \\ 0, & \text { para } w=0 .\end{cases}
$$


Observe que $S$ é linear. De fato, para $h$ e $q$ funções limitadas, $w \geq 1$ e $\alpha \in \mathbb{R}$,

$$
\begin{aligned}
{[S(h+\alpha q)](w) } & =\frac{\mathbb{E}\left[(h+\alpha q)(Z) \mathbf{1}_{\{0,1, \cdots w-1\}}(Z)\right]}{\lambda \mathbb{P}(Z=w-1)} \\
& =\frac{\mathbb{E}\left[h(Z) \mathbf{1}_{\{0,1, \cdots w-1\}}(Z)+\alpha q(Z) \mathbf{1}_{\{0,1, \cdots w-1\}}(Z)\right]}{\lambda \mathbb{P}(Z=w-1)} \\
& =(S h)(w)+\alpha(S q)(w) .
\end{aligned}
$$

Da definição de $S$ temos que

$$
(S h)(w+1)=\frac{\mathbb{E}\left[h(Z) 1_{\{0,1, \cdots, w\}}(Z)\right]}{\lambda \mathbb{P}(Z=w)}, \quad \text { para } w \geq 0 .
$$

Lema $1.8 O$ operador linear $S$ é o inverso do operador $T$, isto é, para todo $h$, $T(S h)=h$.

Prova: Pelas definições dos operadores $S$ e $T$ e do Lema 1.4 segue que:

Para $w=0$,

$$
\begin{aligned}
{[T(S h)](0) } & =\lambda(S h)(1)-0(S h)(0)=\lambda \frac{\mathbb{E}\left[h(Z) 1_{\{0\}}(Z)\right]}{\lambda \mathbb{P}(Z=0)} \\
& =\frac{h(0) \mathbb{P}(Z=0)}{\mathbb{P}(Z=0)}=h(0),
\end{aligned}
$$

e para $w>0$

$$
\begin{aligned}
{[T(S h)](w) } & =\lambda(S h)(w+1)-w(S h)(w) \\
& =\lambda \frac{\mathbb{E}\left[h(Z) \mathbf{1}_{\{0,1, \cdots, w\}}(Z)\right]}{\lambda \mathbb{P}(Z=w)}-w \frac{\mathbb{E}\left[h(Z) \mathbf{1}_{\{0,1, \cdots, w-1\}}(Z)\right]}{\lambda \mathbb{P}(Z=w-1)} \\
& =\frac{\mathbb{E}\left[h(Z) \mathbf{1}_{\{0,1, \cdots, w\}}(Z)\right]}{\mathbb{P}(Z=w)}-\frac{w \mathbb{E}\left[h(Z) \mathbf{1}_{\{0,1, \cdots, w-1\}}(Z)\right]}{w \mathbb{P}(Z=w)} \\
& =\frac{\mathbb{E}\left[h(Z) \mathbf{1}_{\{0,1, \cdots, w-1\}}(Z)\right]}{\mathbb{P}(Z=w)}+\frac{h(w) \mathbb{P}(Z=w)}{\mathbb{P}(Z=w)}- \\
& \frac{\mathbb{E}\left[h(Z) \mathbf{1}_{\{0,1, \cdots, w-1\}}(Z)\right]}{\mathbb{P}(Z=w)}=h(w) .
\end{aligned}
$$


Lema 1.9 Para $A \subset \mathbb{Z}_{+}$arbitrário, a solução da equação (1.1) (de Chen-Stein) com $h(w)=\mathbf{1}_{A}(w)$ pode ser expressa por $f(0)=0$, e para $w \geq 0$ por

$$
f(w+1)=\lambda^{-w-1} w ! e^{\lambda}[\mathbb{P}(Z \in A \cap\{0,1, \ldots, w\})-\mathbb{P}(Z \in A) \mathbb{P}(Z \leq w)],
$$

onde $\mathbb{P}$ denota a probabilidade de uma variável aleatória $P(\lambda)$.

Prova: Definamos para $A \subset \mathbb{Z}_{+}, h_{A}(w)=\mathbf{1}_{A}(w)-\mathbb{P}(Z \in A)$. Temos então que $\mathbb{E}[h(Z)]=0$ e por (1.3), para $w \geq 0$ temos que

$$
\begin{aligned}
(S h)(w+1) & =\frac{\mathbb{E}\left\{\left[\mathbf{1}_{A}(Z)-\mathbb{P}(Z \in A)\right] \mathbf{1}_{\{1,2, \cdots, w\}}(Z)\right\}}{\lambda \mathbb{P}(Z=w)} \\
& =\frac{\mathbb{E}\left\{\mathbf{1}_{A}(Z) \mathbf{1}_{\{1,2, \cdots, w\}}(Z)\right\}-\mathbb{P}(Z \in A) \mathbb{E}\left\{\mathbf{1}_{\{1,2, \cdots, w\}}(Z)\right\}}{\lambda \mathbb{P}(Z=w)} \\
& =\frac{\mathbb{P}(Z \in A \cap\{0,1, \cdots, w\})-\mathbb{P}(Z \in A) \mathbb{P}(Z \leq w)}{\lambda \mathbb{P}(Z=w)} .
\end{aligned}
$$

Como $f(w+1)=(S h)(w+1)$, concluímos que

$f(w+1)=\lambda^{-w-1} w ! e^{\lambda}[\mathbb{P}(Z \in A \cap\{0,1, \ldots, w\})-\mathbb{P}(Z \in A) \mathbb{P}(Z \leq w)], \quad w \geq 0$.

Vamos agora. através de alguns lemas, determinar uma cota superior para $\|f\|$ e $\|\triangle f\|$, onde definimos $\triangle f(w)=f(w+1)-f(w)$ para $w \geq 0$.

Lema 1.10 Para $\lambda>0, w \geq 2$ e $w \geq \lambda-1$ temos que

$$
\frac{(w+2)(w+3)+\lambda}{(w+1)(w+2)(w+3-\lambda)} \leq 1 .
$$

Prova: Como $w+1 \geq \lambda, w+3-\lambda \geq \lambda-1+3-\lambda=2$, e $w+1 \geq 3$ segue que $(w+1)^{-1} \leq \frac{1}{3}$ e $(w+2)^{-1} \leq \frac{1}{4}$, então

$$
\begin{aligned}
& \frac{(w+2)(w+3)+\lambda}{(w+1)(w+2)(w+3-\lambda)} \\
& \quad \leq \frac{(w+2)(w+3)}{(w+1)(w+2)(w+3-\lambda)}+\frac{w+1}{(w+1)(w+2)(w+3-\lambda)} \\
& \quad=\frac{w+3}{(w+1)(w+3-\lambda)}+\frac{1}{(w+2)(w+3-\lambda)}
\end{aligned}
$$




$$
\begin{aligned}
& =\frac{w+1+2}{(w+1)(w+3-\lambda)}+\frac{1}{(w+2)(w+3-\lambda)} \\
& =\frac{w+1}{(w+1)(w+3-\lambda)}+\frac{2}{(w+1)(w+3-\lambda)}+\frac{1}{(w+2)(w+3-\lambda)} \\
& \leq \frac{1}{2}+\frac{2}{2(w+1)}+\frac{1}{2(w+2)} \leq \frac{1}{2}+\frac{1}{3}+\frac{1}{8}=\frac{23}{24} \leq 1 .
\end{aligned}
$$

Lema 1.11 Para $\lambda>0, \frac{1-e^{-\lambda}}{\lambda} \leq 1$.

Prova: Para $\lambda \geq 1$, é imediato. Para $0<\lambda<1$, temos que $\lim _{\lambda \rightarrow 0^{+}} \frac{1-e^{-\lambda}}{\lambda}=1$.

Consideremos $g(\lambda)=1-e^{-\lambda}-\lambda$ para $\lambda \in(0,1)$. Vejamos que $g(\lambda)<0$ para todo $\lambda \in(0,1)$. Temos que $g^{(1)}(\lambda)=e^{-\lambda}-1$. Assim, $g^{(1)}(\lambda)=0$ somente se $\lambda=0$. Além disso, $g^{(1)}(1)<0$ para todo $\lambda \in(0,1)$, logo $g(\lambda)$ é decrescente no intervalo $(0,1)$ e como $g(0)=0$ concluimos que $g(\lambda)<0$ para todo $\lambda \in(0,1)$, isto é $\frac{1-e^{-\lambda}}{\lambda}<1$.

Lema 1.12 Para todo $\lambda>0, w \geq 0$, existe $c_{1}>0$ tal que a solução $f$ da equação (1.1) (de Chen-Stein) satisfaz $\|f\| \leq \min \left(1, c_{1} \lambda^{-\frac{1}{2}}\right)$.

Prova: Para $A \subset \mathbb{Z}_{+}$e denotando o evento $\{Z \leq w\}$ por $U_{w}=\{0,1, \cdots, w\}$ em (1.4) temos que

$$
\begin{aligned}
\mathbb{P}\left(A \cap U_{w}\right)-\mathbb{P}(A) \mathbb{P}\left(U_{w}\right)= & \mathbb{P}\left(A \cap U_{w}\right)-\mathbb{P}\left(A \cap U_{w}\right) \mathbb{P}\left(U_{w}\right) \\
& -\mathbb{P}(A) \mathbb{P}\left(U_{w}\right)+\mathbb{P}\left(A \cap U_{w}\right) \mathbb{P}\left(U_{w}\right) \\
= & \mathbb{P}\left(A \cap U_{w}\right)\left[1-\mathbb{P}\left(U_{w}\right)\right]-\mathbb{P}\left(U_{w}\right)\left[\mathbb{P}(A)-\mathbb{P}\left(A \cap U_{w}\right)\right] \\
= & \mathbb{P}\left(A \cap U_{w}\right) \mathbb{P}\left(U_{w}^{c}\right)-\mathbb{P}\left(A \cap U_{w}^{c}\right) \mathbb{P}\left(U_{w}\right)
\end{aligned}
$$

onde $U_{w}^{c}$ é o complementar de $U_{w}$. Logo (1.4) pode ser escrito para $w \geq 0$ como

$$
f(w+1)=\lambda^{-w-1} w ! e^{\lambda}\left[\mathbb{P}\left(A \cap U_{w}\right) \mathbb{P}\left(U_{w}^{c}\right)-\mathbb{P}\left(A \cap U_{w}^{c}\right) \mathbb{P}\left(U_{w}\right)\right]
$$

Temos que

$$
0 \leq \mathbb{P}\left(A \cap U_{w}\right) \mathbb{P}\left(U_{w}^{c}\right) \leq \mathbb{P}\left(U_{w}\right) \mathbb{P}\left(U_{w}^{c}\right)
$$


e portanto

$$
\begin{aligned}
\mathbb{P}\left(A \cap U_{w}\right)-\mathbb{P}(A) \mathbb{P}\left(U_{w}\right) & \leq \mathbb{P}\left(U_{w}\right) \mathbb{P}\left(U_{w}^{c}\right)-\mathbb{P}\left(U_{w}\right) \mathbb{P}\left(A \cap U_{w}^{c}\right) \\
& =\mathbb{P}\left(U_{w}\right)\left[\mathbb{P}\left(U_{w}^{c}\right)-\mathbb{P}\left(A \cap U_{w}^{c}\right)\right] \\
& =\mathbb{P}\left(U_{w}\right) \mathbb{P}\left(U_{w}^{c} \cap A^{c}\right) \\
& \leq \mathbb{P}\left(U_{w}\right) \mathbb{P}\left(U_{w}^{c}\right) .
\end{aligned}
$$

Para qualquer $A \subset \mathbb{Z}_{+}, w \geq 0$ temos que

$$
|f(w+1)| \leq \lambda^{-w-1} w ! e^{\lambda} \mathbb{P}\left(U_{w}\right) \mathbb{P}\left(U_{w}^{c}\right) .
$$

Agora, para $w<\lambda$,

$$
\begin{aligned}
|f(w+1)| & \leq \lambda^{-w-1} w ! e^{\lambda} \mathbb{P}\left(U_{w}\right)=\lambda^{-w-1} w ! e^{\lambda} \sum_{r=0}^{w} \frac{e^{-\lambda} \lambda^{r}}{r !} \\
& =\lambda^{-w-1} w ! \sum_{r=0}^{w} \frac{\lambda^{r}}{r !}=\lambda^{-w-1} w !\left(\frac{1}{0 !}+\frac{\lambda}{1 !}+\cdots+\frac{\lambda^{w}}{w !}\right) \\
& =\lambda^{-1} w !\left(\frac{\lambda^{-w}}{0 !}+\frac{\lambda^{-w+1}}{1 !}+\cdots+\frac{1}{w !}\right)=\lambda^{-1} w ! \sum_{r=0}^{w} \frac{\lambda^{-r}}{(w-r) !} \\
& =\lambda^{-1} \sum_{r=0}^{w} \frac{\lambda^{-r} w(w-1) \cdots(w-r+1)(w-r) !}{(w-r) !} \\
& \leq \lambda^{-1} \sum_{r=0}^{w}\left(\frac{w}{\lambda}\right)^{r} \leq \lambda^{-1} \sum_{r=0}^{\infty}\left(\frac{w}{\lambda}\right)^{r} \\
& =\lambda^{-1} \frac{\lambda}{\lambda-w}=(\lambda-w)^{-1}
\end{aligned}
$$

e segue que

$$
|f(w+1)| \leq(\lambda-w)^{-1} \leq 1, \text { quando } w \leq \lambda-1 .
$$

Para $w>\lambda-3$,

$$
\begin{aligned}
|f(w+1)| & \leq \lambda^{-w-1} w ! e^{\lambda} \mathbb{P}\left(U_{w}^{c}\right)=\lambda^{-w-1} w ! e^{\lambda} \sum_{r=w+1}^{\infty} \frac{e^{-\lambda} \lambda^{r}}{r !} \\
& =w ! \sum_{r=w+1}^{\infty} \frac{\lambda^{r-w-1}}{r !}=w ! \sum_{j=0}^{\infty} \frac{\lambda^{j}}{(j+w+1) !} \\
& =\frac{w !}{(w+1) !}+\frac{w ! \lambda}{(w+2) !}+\cdots+\frac{w ! \lambda^{l}}{(w+l+1) !}+\cdots
\end{aligned}
$$




$$
\begin{aligned}
& =\frac{1}{w+1}+\frac{\lambda}{(w+1)(w+2)}+\cdots+\frac{\lambda^{l}}{(w+1) \cdots(w+l+1)}+\cdots \\
& =\frac{1}{w+1}+\frac{\lambda}{(w+1)(w+2)}\left[1+\frac{\lambda}{w+3}+\frac{\lambda^{2}}{(w+3)(w+4)}+\cdots\right] \\
& \leq \frac{1}{w+1}+\frac{\lambda}{(w+1)(w+2)}\left[1+\frac{\lambda}{w+3}+\frac{\lambda^{2}}{(w+3)^{2}}+\cdots\right] \\
& =\frac{1}{w+1}+\frac{\lambda}{(w+1)(w+2)} \sum_{t=0}^{\infty}\left(\frac{\lambda}{w+3}\right)^{t} \\
& =\frac{1}{w+1}\left[1+\frac{\lambda}{(w+2)} \sum_{t=0}^{\infty}\left(\frac{\lambda}{w+3}\right)^{t}\right] \\
& =\frac{1}{w+1}\left[1+\frac{\lambda}{(w+2)} \frac{w+3}{(w+3-\lambda)}\right] \\
& =\frac{1}{w+1}\left[\frac{(w+2)(w+3-\lambda)+\lambda(w+3)}{(w+2)(w+3-\lambda)}\right] \\
& =\frac{1}{w+1}\left[\frac{(w+2)(w+3)-\lambda(w+2)+\lambda(w+3)}{(w+2)(w+3-\lambda)}\right] \\
& =\frac{(w+1)(w+2)(w+3-\lambda)}{w+3)+\lambda}
\end{aligned}
$$

De (1.8) segue que

$$
\frac{(w+2)(w+3)+\lambda}{(w+1)(w+2)(w+3-\lambda)} \leq 1
$$

quando

$$
\begin{aligned}
(w+2)(w+3)+\lambda & \leq(w+1)(w+2)(w+3-\lambda) \\
\lambda & \leq(w+1)(w+2)(w+3-\lambda)-(w+2)(w+3) \\
3 \lambda+\lambda w(w+3) & \leq w\left(w^{2}+5 w+6\right) \\
\lambda & \leq \frac{w(w+3)(w+2)}{3+w(w+3)} .
\end{aligned}
$$

Logo, para $w=1$ e $\lambda \leq \frac{12}{7}$ temos $|f(w+1)| \leq 1$. Para $w \geq 2$ e $w \geq \lambda-1$ pelo Lema 1.10 temos que $|f(w+1)| \leq 1$. 
Considerando agora $w=0$ com $0<\lambda \leq 1$, de (1.6) temos que

$$
|f(1)| \leq \lambda^{-1} e^{\lambda} \mathbb{P}\left(U_{0}\right) \mathbb{P}\left(U_{0}^{c}\right)=\lambda^{-1} e^{\lambda} e^{-\lambda}\left(1-e^{-\lambda}\right)=\frac{1-e^{-\lambda}}{\lambda}
$$

e pelo Lema 1.11 temos que $\frac{1-e^{-\lambda}}{\lambda} \leq 1$ para todo $\lambda>0$, logo $|f(1)| \leq 1$.

Para $w=1$ e $\frac{12}{7} \leq \lambda \leq 2$

$$
\begin{aligned}
|f(2)| & \leq \lambda^{-2} e^{\lambda} \mathbb{P}\left(U_{1}\right) \mathbb{P}\left(U_{1}^{c}\right) \\
& =\lambda^{-2} e^{\lambda}\left(e^{-\lambda}+\lambda e^{-\lambda}\right)\left(1-e^{-\lambda}-\lambda e^{-\lambda}\right) \\
& =\left\{\lambda^{-2}(1+\lambda)\right\}\left\{1-e^{-\lambda}(1+\lambda)\right\},
\end{aligned}
$$

de onde segue que os dois termos são monótonos em $\lambda$. De fato, para $l(\lambda)=$ $\lambda^{-2}(1+\lambda)$ temos que $l^{(1)}(\lambda)=\frac{\lambda^{2}-2 \lambda-2 \lambda^{2}}{\lambda^{4}}=-\frac{\lambda+2}{\lambda^{3}} \leq 0$ e portanto $l(\lambda)$ é monótono decrescente. Para $m(\lambda)=1-e^{-\lambda}(1+\lambda), m^{(1)}(\lambda)=-\left(e^{-\lambda}-e^{-\lambda}(1+\lambda)\right)=$ $-e^{-\lambda}(1-1-\lambda)=\lambda e^{-\lambda} \geq 0$ para todo $\lambda \geq 0$ e segue que $m(\lambda)$ é monótona crescente. Portanto

$$
|f(2)| \leq\left\{\frac{133}{144}\right\}\left\{1-3 e^{-2}\right\}<1,
$$

e segue que $|f(w+1)| \leq 1$.

Usando a fórmula de Stirling, $w ! \leq \sqrt{2 \pi} w^{w+\frac{1}{2}} \exp \left(-w+\frac{1}{12 w}\right), w \geq 0$ e como $\mathbb{P}\left(U_{w}\right) \mathbb{P}\left(U_{w}^{c}\right) \leq \frac{1}{4}$, segue então de (1.6) que

$$
\begin{aligned}
|f(w+1)| & \leq \frac{1}{4} \sqrt{2 \pi} \lambda^{-w-1} e^{\lambda} w^{w+\frac{1}{2}} \exp \left(-w+\frac{1}{12 w}\right) \\
& =\frac{1}{4} \sqrt{2 \pi} \lambda^{-\frac{1}{2}}\left(\frac{w}{\lambda}\right)^{w+\frac{1}{2}} \exp \left(\lambda-w+\frac{1}{12 w}\right) \\
& =\frac{1}{4} \sqrt{2 \pi} \lambda^{-\frac{1}{2}} \exp \left\{\lambda-w+\frac{1}{12 w}+\left(w+\frac{1}{2}\right) \ln \left(\frac{w}{\lambda}\right)\right\} .
\end{aligned}
$$

Como $\ln (x) \leq x-1$ para $x>0$, temos para $w \geq 1$ 


$$
\begin{aligned}
|f(w+1)| & \leq \frac{1}{4} \sqrt{2 \pi} \lambda^{-\frac{1}{2}} \exp \left\{\lambda-w+\frac{1}{12 w}+\left(w+\frac{1}{2}\right)\left(\frac{w}{\lambda}-1\right)\right\} \\
& =\frac{1}{4} \sqrt{2 \pi} \lambda^{-\frac{1}{2}} \exp \left\{\lambda-w+\frac{1}{12 w}+\lambda^{-1}\left(w+\frac{1}{2}\right)(w-\lambda)\right\} \\
& =\frac{1}{4} \sqrt{2 \pi} \lambda^{-\frac{1}{2}} \exp \left\{(w-\lambda)\left[\lambda^{-1}\left(w+\frac{1}{2}\right)-1\right]+\frac{1}{12 w}\right\} \\
& =\frac{1}{4} \sqrt{2 \pi} \lambda^{-\frac{1}{2}} \exp \left\{\lambda^{-1}(w-\lambda)\left(w+\frac{1}{2}-\lambda\right)+\frac{1}{12 w}\right\} .
\end{aligned}
$$

Sabemos que $|f(1)| \leq \frac{1-e^{-\lambda}}{\lambda}$ e $1-e^{-\lambda} \leq 1$ para todo $\lambda$ então $|f(1)| \leq \lambda^{-1}$. Comparando (1.7) com (1.8) e (1.10) e tomando o menor segue que $\|f\| \leq c_{1} \lambda^{-\frac{1}{2}}$. Logo, tomando $\min \left(1, c_{1} \lambda^{-\frac{1}{2}}\right)$ segue que $\|f\| \leq \min \left(1, c_{1} \lambda^{-\frac{1}{2}}\right)$.

Observação 1.2: A aproximação de Stirling dada por

$$
n ! \sim \sqrt{2 \pi} n^{n+\frac{1}{2}} e^{-n}
$$

e usada em (1.9) é bem conhecida. O sinal é usado para indicar que o quociente de um lado pelo outro converge a 1 quando $n$ vai ao infinito. O erro percentual que se comete ao utilizarmos a aproximação decresce regularmente e a precisão é notável, mesmo para valores pequenos de $n$. Em [10] encontramos uma prova para a aproximação dada por

$$
\sqrt{2 \pi} n^{n+\frac{1}{2}} e^{-n} e^{(12 n+1)^{-1}}<n !<\sqrt{2 \pi} n^{n+\frac{1}{2}} e^{-n} e^{(12 n)^{-1}}
$$

onde o quociente dos membros extremos está próximo de $1-\left(12 n^{2}\right)^{-1}$ e portanto, o lado direito das desigualdades sobrestima $n$ !, mas com um erro inferior a $9 n^{-2}$ por cento.

Para o lema a seguir, adotaremos a seguinte notação, $f_{j}$ é a função solução da equação (1.1) (de Chen-Stein), que depende do parâmetro $\lambda$, quando restrita ao conjunto $\{j\} \subset \mathbb{Z}_{+}$, isto é, $f_{j}(w)=f(w) 1_{\{j\}}(w)$, para todo $w \geq 0$. 
Lema 1.13 Para todo $\lambda>0, w \geq 0$, a solução $f$ da equação (1.1) (de ChenStein) satisfaz $\|\triangle f\| \leq \frac{1-e^{-\lambda}}{\lambda}$.

Prova: Seja $f_{j}$ denotando $f_{\lambda,\{j\}}$ e notemos que de $(1.5), f_{\lambda, A}(w+1)=\sum_{j \in A} f_{j}$ para todo conjunto $A \subset \mathbb{Z}_{+}$.

De fato, seja $\{j\}=A, A \subset \mathbb{Z}_{+}$e $U_{w}$ definido na demonstração do Lema 1.12. Se $w \geq j, A \cap \mathcal{L}_{w}^{*}=\{j\}$ e portanto

$$
\mathbb{P}\left(A \cap U_{w}\right)=\mathbb{P}(\{j\})=\frac{e^{-\lambda} \lambda^{j}}{j !} \quad \text { e } \mathbb{P}\left(A \cap U_{w}^{c}\right)=\mathbb{P}(\emptyset)=0
$$

onde $\emptyset$ denota o conjunto vazio, logo usando (1.5) temos que

$$
f_{j}(w+1)=\lambda^{-w-1} w ! e^{\lambda}\left[\frac{e^{-\lambda} \lambda^{j}}{j !} \mathbb{P}\left(U_{w}^{c}\right)-0\right]=\frac{\lambda^{j-1}}{j !} w ! \lambda^{-w} \mathbb{P}\left(U_{w}^{c}\right) .
$$

Se $w<j$, então $A \cap U_{w}^{c}=\{j\}$ e portanto

$$
\mathbb{P}\left(A \cap U_{w}^{c}\right)=\mathbb{P}(\{j\})=\frac{e^{-\lambda} \lambda^{j}}{j !} \quad \text { e } \quad \mathbb{P}\left(A \cap U_{w}\right)=\mathbb{P}(\emptyset)=0
$$

e de novo por (1.j) temos que

$$
f_{j}(w+1)=-\frac{\lambda^{j-1}}{j !} w ! \lambda^{-w} \mathbb{P}\left(U_{w}\right)
$$

Segue que

$$
f_{j}(w+1)=\left\{\begin{array}{cc}
\frac{\lambda^{\jmath-1}}{j !} w ! \lambda^{-w} \mathbb{P}\left(U_{w}^{c}\right), & \text { se } w \geq j \\
-\frac{\lambda^{\jmath-1}}{j !} w ! \lambda^{-w} \mathbb{P}\left(U_{w}\right), & \text { se } w<j
\end{array}\right.
$$

Como vale para todo elemento de $A$, concluímos que $f_{\lambda, A}(w+1)=\sum_{j \in A} f_{j}$ e notamos que $f_{j}$ é positiva e decrescente em $w \geq j+1$ e é negativa e decrescente em $w \leq j$. Daí, $f_{j}(w+1)-f_{j}(w)$ só toma o valor positivo em $j=w$. Temos

$$
\begin{aligned}
f_{j}(j+1)-f_{j}(j)= & \frac{\lambda^{j-1}}{j !} j ! \lambda^{-j} \mathbb{P}(Z \geq j+1) \\
& -\left[-\frac{\lambda^{j-1}}{j !}(j-1) ! \lambda^{-j+1} \mathbb{P}(Z \leq j-1)\right]
\end{aligned}
$$




$$
\begin{aligned}
& =\lambda^{-1} \sum_{r=j+1}^{\infty} \frac{e^{-\lambda} \lambda^{r}}{r !}+\frac{1}{j} \sum_{r=0}^{j-1} \frac{e^{-\lambda} \lambda^{r}}{r !} \\
& =\lambda^{-1} \sum_{r=j+1}^{\infty} \frac{e^{-\lambda} \lambda^{r}}{r !}+\frac{1}{j} \sum_{r=1}^{j} \frac{e^{-\lambda} \lambda^{r-1} r}{(r-1) ! r} \\
& =\lambda^{-1} e^{-\lambda}\left[\sum_{r=j+1}^{\infty} \frac{\lambda^{r}}{r !}+\sum_{r=1}^{j} \frac{\lambda^{r}}{r !} \frac{r}{j}\right]
\end{aligned}
$$

Para $r \leq j, \sum_{r=1}^{j} \frac{\lambda^{r}}{r !} \frac{r}{j} \leq \sum_{r=1}^{j} \frac{\lambda^{r}}{r !}$, e portanto

$$
\begin{aligned}
f_{j}(j+1)-f_{j}(j) & \leq \lambda^{-1} e^{-\lambda}\left[\sum_{r=j+1}^{\infty} \frac{\lambda^{r}}{r !}+\sum_{r=1}^{j} \frac{\lambda^{r}}{r^{!}}\right] \\
& =\lambda^{-1} e^{-\lambda}\left(e^{\lambda}-1\right) \\
& =\lambda^{-1}\left(1-e^{-\lambda}\right) .
\end{aligned}
$$

Assim, para todo $A \subset \mathbb{Z}_{+}$,

$$
f_{\lambda, A}(w+1)-f_{\lambda, A}(w)=\mathbf{1}_{A}(w)\left\{f_{w}(w+1)-f_{w}(w)\right\}+\sum_{\substack{j \in A \\ j \neq w}}\left\{f_{j}(w+1)-f_{j}(w)\right\}
$$

onde só o primeiro termo é positivo. Segue que

$$
\sup _{w \in \mathbb{Z}_{+}}\left\{f_{\lambda, A}(w+1)-f_{\lambda, A}(w)\right\}=\sup _{w \in \mathbb{Z}_{+}}\left\{f_{w}(w+1)-f_{w}(w)\right\} \leq \lambda^{-1}\left(1-e^{-\lambda}\right) .
$$

Finalmente, vejamos que $f_{\lambda, A}=-f_{\lambda, A^{c}}$ o que completa a prova. Com efeito,

$$
\begin{aligned}
f_{\lambda, A}(w+1)= & \lambda^{-w-1} w ! e^{\lambda}\left[\mathbb{P}\left(A \cap U_{w}\right) \mathbb{P}\left(U_{w}^{c}\right)-\mathbb{P}\left(A \cap U_{w}^{c}\right) \mathbb{P}\left(U_{w}\right)\right] \\
= & \lambda^{-w-1} w ! e^{\lambda}\left[\mathbb{P}\left(U_{w}-A^{c}\right) \mathbb{P}\left(U_{w}^{c}\right)-\mathbb{P}\left(U_{w}^{c}-A^{c}\right) \mathbb{P}\left(U_{w}\right)\right] \\
= & \lambda^{-w-1} w ! e^{\lambda}\left\{\left[\mathbb{P}\left(U_{w}\right)-\mathbb{P}\left(U_{w} \cap A^{c}\right)\right] \mathbb{P}\left(U_{w}^{c}\right)\right. \\
& \left.-\left[\mathbb{P}\left(U_{w}^{c}\right)-\mathbb{P}\left(U_{w}^{c} \cap A^{c}\right)\right] \mathbb{P}\left(U_{w}\right)\right\} \\
= & \lambda^{-w-1} w ! e^{\lambda}\left[\mathbb{P}\left(U_{w}\right) \mathbb{P}\left(U_{w}^{c}\right)-\mathbb{P}\left(U_{w} \cap A^{c}\right) \mathbb{P}\left(U_{w}^{c}\right)\right. \\
& \left.-\mathbb{P}\left(U_{w}^{c}\right) \mathbb{P}\left(U_{w}\right)+\mathbb{P}\left(U_{w}^{c} \cap A^{c}\right) \mathbb{P}\left(U_{w}\right)\right] \\
= & -\lambda^{-w-1} w ! e^{\lambda}\left[\mathbb{P}\left(U_{w} \cap A^{c}\right) \mathbb{P}\left(U_{w}^{c}\right)-\mathbb{P}\left(U_{w}^{c} \cap A^{c}\right) \mathbb{P}\left(U_{w}\right)\right] \\
= & -f_{\lambda, A^{c}}(w+1) .
\end{aligned}
$$


Portanto, temos que

$$
f_{\lambda, A}(w)-f_{\lambda, A}(w+1)=f_{\lambda, A^{c}}(w+1)-f_{\lambda, A^{c}}(w) \leq \lambda^{-1}\left(1-e^{-\lambda}\right) .
$$

Lema 1.14 Para todo $\lambda>0, w \geq 0$, e $h_{\{0\}}(w)=1_{\{0\}}(w)-e^{-\lambda}$, temos que a solução $f$ da equação (1.1) (de Chen-Stein) satisfaz $\|f\|=\frac{1-e^{-\lambda}}{\lambda}$.

Prova: Como $\mathbb{E}[h(Z)]=0$ então o operador $S$ pode ser escrito como

$$
(S h)(w+1)=\frac{\mathbb{P}(Z \leq \min (0, w))-\mathbb{P}(Z=0) \mathbb{P}(Z \leq w)}{\lambda \mathbb{P}(Z \leq w)}, \quad \text { para } w \geq 0
$$

Temos que

$$
\begin{aligned}
\frac{d}{d \lambda} \mathbb{P}(Z \leq j) & =\frac{d}{d \lambda}\left[\sum_{z=0}^{j} \frac{e^{-\lambda} \lambda^{z}}{z !}\right]=\sum_{z=0}^{j} \frac{d}{d \lambda} \frac{e^{-\lambda} \lambda^{z}}{z !} \\
& =\sum_{z=0}^{j} \frac{1}{z !}\left[z e^{-\lambda} \lambda^{z-1}-\lambda^{z} e^{-\lambda}\right]=\sum_{z=1}^{j} \frac{e^{-\lambda} \lambda^{z-1}}{(z-1) !}-\sum_{z=0}^{j} \frac{e^{-\lambda} \lambda^{z}}{z !} \\
& =\sum_{l=0}^{j-1} \frac{e^{-\lambda} \lambda^{l}}{l !}-\sum_{l=0}^{j} \frac{e^{-\lambda} \lambda^{l}}{l !}=-\frac{e^{-\lambda} \lambda^{j}}{j !}=-\mathbb{P}(Z=j) .
\end{aligned}
$$

Também, $\mathbb{P}(Z \leq j)$ pode ser escrita como

$$
\begin{aligned}
\mathbb{P}(Z \leq j) & =\sum_{z=0}^{j} \frac{e^{-\lambda} \lambda^{z}}{z !}=\sum_{z=0}^{j} \frac{e^{-\lambda} \lambda^{j-z}}{(j-z) !} \\
& =\sum_{z=0}^{j} \frac{j !}{z !(j-z) !} \frac{z !}{j !} e^{-\lambda} \lambda^{j-z}=\sum_{z=0}^{j}\left(\begin{array}{l}
j \\
z
\end{array}\right) \frac{z !}{j !} e^{-\lambda} \lambda^{j-z}
\end{aligned}
$$

e por definição de $z !=\int_{0}^{\infty} t^{z} e^{-t} d t$ temos que

$$
\begin{aligned}
\mathbb{P}(Z \leq j) & =\sum_{z=0}^{j}\left(\begin{array}{l}
j \\
z
\end{array}\right) \frac{e^{-\lambda} \lambda^{j-z}}{j !} \int_{0}^{\infty} t^{z} e^{-t} d t \\
& =\int_{0}^{\infty} \frac{1}{j !} \sum_{z=0}^{j}\left(\begin{array}{l}
j \\
z
\end{array}\right) e^{-\lambda} \lambda^{j-z} t^{z} e^{-t} d t
\end{aligned}
$$




$$
\begin{aligned}
& =\int_{0}^{\infty} \frac{1}{j !} e^{-(\lambda+t)} \sum_{z=0}^{j}\left(\begin{array}{l}
j \\
z
\end{array}\right) \lambda^{j-z} t^{z} d t \\
& =\int_{0}^{\infty} \frac{1}{j !} e^{-(\lambda+t)}(\lambda+t)^{j} d t \\
& =\int_{\lambda}^{\infty} \frac{1}{j !} e^{-v} v^{j} d v
\end{aligned}
$$

onde $v=\lambda+t$. Então

$$
\mathbb{P}(Z \leq j)=\int_{\lambda}^{\infty} \frac{1}{j !} e^{-v} v^{j} d v=1-\int_{0}^{\lambda} \frac{e^{-v} v^{j}}{j !} d v .
$$

Usando o Lema $1.9 f(w+1)=(S h)(w+1)$ e portanto, para $w \geq 1$

$$
\begin{aligned}
f(w) & =\frac{\mathbb{P}(Z \leq \min (0, w-1))-\mathbb{P}(Z \leq 0) \mathbb{P}(Z \leq w-1)}{\lambda \mathbb{P}(Z \leq w-1)} \\
& =\frac{\mathbb{P}(Z=0)-\mathbb{P}(Z=0) \mathbb{P}(Z \leq w-1)}{\lambda \mathbb{P}(Z=w-1)} \\
& =\frac{\mathbb{P}(Z=0)[1-\mathbb{P}(Z \leq w-1)]}{\lambda \mathbb{P}(Z=w-1)}=\frac{e^{-\lambda} \mathbb{P}(Z \geq w)}{\lambda \mathbb{P}(Z=w-1)} .
\end{aligned}
$$

Segue que

$$
f(1)=\frac{e^{-\lambda}\left(1-e^{-\lambda}\right)}{\lambda e^{-\lambda}}=\frac{1-e^{-\lambda}}{\lambda}
$$

e

$$
f(2)=\frac{e^{-\lambda}\left(1-e^{-\lambda}-\lambda e^{-\lambda}\right)}{\lambda^{2} e^{-\lambda}}=\frac{1-e^{-\lambda}}{\lambda^{2}}-\frac{e^{-\lambda}}{\lambda}=f(1) \frac{1}{\lambda}-\frac{e^{-\lambda}}{\lambda},
$$

o que permite concluir que $f(1)>f(2)>\cdots>0$.

$$
\text { Portanto } \sup _{w \geq 0}|f(w)|=f(1)=\frac{1-e^{-\lambda}}{\lambda} \text {. }
$$

Definindo $h_{A}(w)=\mathbf{1}_{A}(w)$ para subconjuntos $A \subset \mathbb{Z}_{+}$, e tomando a esperança matemática em ambos os lados de (1.1) temos que

$$
\mathbb{E}[\lambda f(W+1)-W f(W)]=\mathbb{E}\left[\mathbf{1}_{A}(W)\right]-\mathbb{E}\left[\mathbf{1}_{A}(Z)\right]=\mathbb{P}(W \in A)-\mathbb{P}(Z \in A)
$$


Para $A \subset \mathbb{Z}_{+}$sabemos que a função solução da equação de Chen-Stein, digamos $f_{A}$, permite escrever a distância em variação total da distribuição de $W$ e $Z \sim P(\lambda)$ por

$$
\begin{aligned}
d_{V T}(W, Z) & =\sup _{A \subset \mathbb{Z}_{+}}|\mathbb{P}(W \in A)-\mathbb{P}(Z \in A)| \\
& =\sup _{A \subset \mathbb{Z}_{+}}\left|\mathbb{E}\left[\lambda f_{A}(W+1)-W f_{A}(W)\right]\right| .
\end{aligned}
$$

Assim, para toda função $h: \mathbb{Z}_{+} \rightarrow \mathbb{R}$ limitada tal que $\|h\|=1$ e $f_{h}$ a respectiva função solução de (1.1), temos que a distância em variação total entre as distribuições de $W$ e $Z$ é

$$
\begin{aligned}
d_{V T}(W, Z) & =\sup _{\|h\|=1}|\mathbb{E}[h(W)]-\mathbb{E}[h(Z)]| \\
& =\sup _{\|h\|=1}\left|\mathbb{E}\left[\lambda f_{h}(W+1)-W f_{h}(W)\right]\right| .
\end{aligned}
$$

No que segue, consideremos $h \operatorname{com}\|h\|=1, f(\cdot)=S(h(\cdot)-\mathbb{E}[h(Z)])$ tal que $(T f)(\cdot)=h(\cdot)-\mathbb{E}[h(Z)]$ onde $\mathbb{E}[(T f)(W)]=\mathbb{E}[h(W)-h(Z)]$. Além disso, seja $X_{\alpha}$ variável aleatória de Bernoulli, $V_{\alpha}=\sum_{\beta \in \Gamma-B_{\alpha}} X_{\beta}, W_{\alpha}=W-X_{\alpha}$, onde $W=\sum_{\alpha \in \Gamma} X_{\alpha}$ tal que $V_{\alpha} \leq W_{\alpha} \leq W$.

Lema 1.15 Seja $\Gamma$ um conjunto índice, $X_{\alpha}$ variável aleatória com distribuição Bernoulli $e \alpha \in \Gamma$ Então:

$$
\begin{aligned}
& \text { a) } X_{\alpha} f(W)=X_{\alpha} f\left(W_{\alpha}+1\right) . \\
& \text { b) } f\left(W_{\alpha}+1\right)-f(W+1)=X_{\alpha}\left[f\left(W_{\alpha}+1\right)-f\left(W_{\alpha}+2\right)\right] .
\end{aligned}
$$

Prova: a) Se $X_{\alpha}=0$, o resultado é imediato.

Se $X_{\alpha}=1$, então $f(W)=f\left(W_{\alpha}+1\right)$.

b) Se $X_{\alpha}=0, f\left(W_{\alpha}+1\right)-f(W+1)=X_{\alpha}\left[f\left(W_{\alpha}+1\right)-f\left(W_{\alpha}+2\right)\right]=0$.

Se $X_{\alpha}=1, f\left(W_{\alpha}+1\right)-f(W+1)=f\left(W_{\alpha}+1\right)-f\left(W_{\alpha}+2\right)$.

Lema 1.16 Para $X_{\alpha}, W_{\alpha}$ e $V_{\alpha}$ variáveis aleatórias, definidas acima,

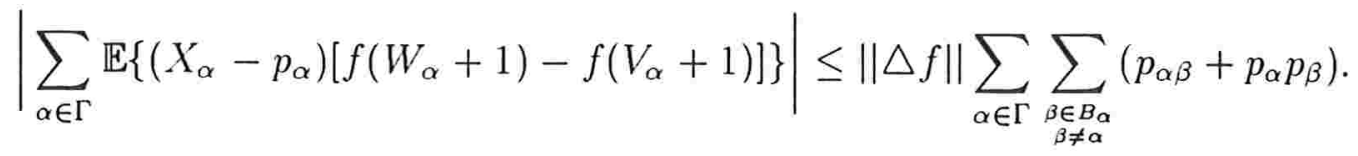


Prova:

$$
\begin{aligned}
& \sum_{\alpha \in \Gamma} \mathbb{E}\left\{\left(X_{\alpha}-p_{\alpha}\right)\left[f\left(W_{\alpha}+1\right)-f\left(V_{\alpha}+1\right)\right]\right\} \\
& =\left|\sum_{\alpha \in \Gamma} \mathbb{E}\left\{\left(X_{\alpha}-p_{\alpha}\right)\left[f\left(\sum_{\substack{3 \in B_{\alpha} \\
\beta \neq \alpha}} X_{\beta}+V_{\alpha}+1\right)-f\left(V_{\alpha}+1\right)\right]\right\}\right| \\
& =\mid \sum_{\alpha \in \Gamma} \mathbb{E}\left\{( X _ { \alpha } - p _ { \alpha } ) \left[f\left(\sum_{\substack{\beta \in B_{\alpha} \\
\beta \neq \alpha, \gamma_{1}}} X_{\beta}+X_{\gamma_{1}}+V_{\alpha}+1\right)-\right.\right. \\
& \left.\left.f\left(\sum_{\substack{\beta \in B_{\alpha} \\
\beta \neq \alpha, \gamma_{1}}} X_{\beta}+V_{\alpha}+1\right)+f\left(\sum_{\substack{\beta \in B_{\alpha} \\
\beta \neq \alpha, \gamma_{1}}} X_{\beta}+V_{\alpha}+1\right)-f\left(V_{\alpha}+1\right)\right]\right\} \mid \\
& =\mid \sum_{\alpha \in \Gamma} \mathbb{E}\left\{( X _ { \alpha } - p _ { \alpha } ) \left[X_{\gamma_{1}}\left(f\left(\sum_{\substack{\beta \in B_{\alpha} \\
\beta \neq \alpha, \gamma_{1}}} X_{\beta}+V_{\alpha}+2\right)-f\left(\sum_{\substack{\beta \in B_{\alpha} \\
\beta \neq \alpha, \gamma_{1}}} X_{\beta}+V_{\alpha}+1\right)\right)\right.\right. \\
& \left.\left.+f\left(\sum_{\substack{\beta \in B_{\alpha} \\
\beta \neq \alpha, \gamma_{1}, \gamma_{2}}} X_{\beta}+X_{\gamma_{2}}+V_{\alpha}+1\right)-f\left(V_{\alpha}+1\right)\right]\right\} \mid \\
& \leq \sum_{\alpha \in \Gamma} \mathbb{E}\left\{\left(X_{\alpha}+p_{\alpha}\right) \mid\left[X_{\gamma_{1}}\left(f\left(\sum_{\substack{\beta \in B_{\alpha} \\
\beta \neq \alpha, \gamma_{1}}} X_{\beta}+V_{\alpha}+2\right)-f\left(\sum_{\substack{\beta \in B_{\alpha} \\
\beta \neq \alpha, \gamma_{1}}} X_{\beta}+V_{\alpha}+1\right)\right) \mid\right.\right. \\
& \left.\left.+f\left(\sum_{\substack{\beta \in B_{\alpha} \\
\beta \neq \alpha, \gamma_{1}, \gamma_{2}}} X_{\beta}+X_{\gamma_{2}}+V_{\alpha}+1\right)-f\left(V_{\alpha}+1\right)\right]\right\} \mid \\
& \leq \sum_{\alpha \in \Gamma} \mathbb{E}\left\{( X _ { \alpha } + p _ { \alpha } ) \left[X_{\gamma_{1}}\|\Delta f\|+\mid f\left(\sum_{\substack{\beta \in B_{\alpha} \\
\beta \neq \alpha, \gamma_{1}, \gamma_{2}}} X_{\beta}+X_{\gamma_{2}}+V_{\alpha}+1\right)-\right.\right. \\
& \left.\left.f\left(\sum_{\substack{\beta \in B_{\alpha} \\
\beta \neq \alpha, \gamma_{1}, \gamma_{2}}} X_{\beta}+V_{\alpha}+1\right)+f\left(\sum_{\substack{\beta \in B_{\alpha} \\
\beta \neq \alpha, \gamma_{1}, \gamma_{2}}} X_{\beta}+V_{\alpha}+1\right)-f\left(V_{\alpha}+1\right)\right] \mid\right\} \\
& \leq \sum_{\alpha \in \Gamma} \mathbb{E}\left\{\left(X_{\alpha}+p_{\alpha}\right)\left[X_{\gamma_{1}}\|\triangle f\|+X_{\gamma_{2}}\|\triangle f\|+\cdots+X_{\gamma_{\left|B_{\alpha}\right|-1}}\|\triangle f\|\right]\right\} \\
& =\sum_{\alpha \in \Gamma} \sum_{\substack{\beta \in B_{\alpha} \\
\beta \neq \alpha}} \mathbb{E}\left\{\left(X_{\alpha}+p_{\alpha}\right) X_{\beta}\|\triangle f\|\right\} \\
& =\|\triangle f\| \sum_{\alpha \in \Gamma} \sum_{\substack{\beta \in B_{\alpha} \\
\beta \neq \alpha}}\left(p_{\alpha \beta}+p_{\alpha} p_{\beta}\right) .
\end{aligned}
$$


Portanto

$$
\left|\sum_{\alpha \in \Gamma} \mathbb{E}\left\{\left(X_{\alpha}-p_{\alpha}\right)\left[f\left(W_{\alpha}+1\right)-f\left(V_{\alpha}+1\right)\right]\right\}\right| \leq\|\triangle f\| \sum_{\alpha \in \Gamma} \sum_{\substack{\beta \in B_{\alpha} \\ \beta \neq ⿱ 亠 乂}}\left(p_{\alpha \beta}+p_{\alpha} p_{\beta}\right) .
$$

Lema 1.17 Para $X_{\alpha}, W_{\alpha}$ e $V_{\alpha}$ variáveis aleatórias, definidas acima,

$$
\left|\sum_{\alpha \in \Gamma} \mathbb{E}\left\{p_{\alpha} X_{\alpha}\left[f\left(W_{\alpha}+1\right)-f\left(W_{\alpha}+2\right)\right]\right\}\right| \leq\|\triangle f\| \sum_{\alpha \in \Gamma} p_{\alpha}^{2} .
$$

Prova:

$$
\begin{aligned}
\left|\sum_{\alpha \in \Gamma} \mathbb{E}\left\{p_{\alpha} X_{\alpha}\left[f\left(W_{\alpha}+1\right)-f\left(W_{\alpha}+2\right)\right]\right\}\right| & \leq \sum_{\alpha \in \Gamma} \mathbb{E}\left[p_{\alpha} X_{\alpha}\left|f\left(W_{\alpha}+2\right)-f\left(W_{\alpha}+1\right)\right|\right] \\
& \leq\|\triangle f\| \sum_{\alpha \in \Gamma} p_{\alpha} \mathbb{E}\left(X_{\alpha}\right) \\
& =\|\triangle f\| \sum_{\alpha \in \Gamma} p_{\alpha}^{2} .
\end{aligned}
$$

Lema 1.18 Para $X_{\alpha}, W_{\alpha}$ e $V_{\alpha}$ variáveis aleatórias, definidas acima,

$$
\left|\sum_{\alpha \in \Gamma} \mathbb{E}\left[\left(X_{\alpha}-p_{\alpha}\right) f\left(V_{\alpha}+1\right)\right]\right| \leq b_{3}|| f \| \text {. }
$$

\section{Prova:}

$$
\begin{aligned}
\left|\mathbb{E}\left\{\left(X_{\alpha}-p_{\alpha}\right) f\left(V_{\alpha}+1\right)\right\}\right| & =\left|\mathbb{E}\left\{\mathbb{E}\left[\left(X_{\alpha}-p_{\alpha}\right) f\left(V_{\alpha}+1\right) / V_{\alpha}\right]\right\}\right| \\
& \leq \mathbb{E}\left|f\left(V_{\alpha}+1\right) \mathbb{E}\left\{\left(X_{\alpha}-p_{\alpha}\right) / V_{\alpha}\right\}\right| \\
& \leq|| f|| \mathbb{E}\left|\mathbb{E}\left\{\left(X_{\alpha}-p_{\alpha}\right) / V_{\alpha}\right\}\right|=s_{\alpha}\|f\| .
\end{aligned}
$$

A prova do Teorema 1.2, dada a seguir, apresenta o resultado em que se os valores de $b_{1}, b_{2}$ e $b_{3}\left(b_{3}^{\prime}\right)$ são pequenos então o número total de eventos $W$ é aproximadamente Poisson e determina uma cota para a velocidade de convergência.

Prova do Teorema 1.2: Seja $h$ fixada com $\|h\|=1$ e $f(\cdot)=S(h(\cdot)-\mathbb{E}[h(Z)])$. Pela equação (1.1) (de Chen-Stein) temos que

$$
\lambda f(w+1)-w f(w)=(T f)(w)=h(w)-\mathbb{E} h(Z)
$$


e portanto onde $\mathbb{E}[(T f)(W)]=\mathbb{E}[h(W)-h(Z)]$. Escrevendo $V_{\alpha}=\sum_{\beta \in B_{\alpha}^{c}} X_{\beta}$ e $W_{\alpha}=W-X_{\alpha}$, segue que $V_{\alpha} \leq W_{\alpha} \leq W$, e pelo Lema 1.15 temos que $X_{\alpha} f(W)=X_{\alpha} f\left(W_{\alpha}+1\right)$ e $f\left(W_{\alpha}+1\right)-f(W+1)=X_{\alpha}\left[f\left(W_{\alpha}+1\right)-f\left(W_{\alpha}+2\right)\right]$. Logo, aplicando (1.12) temos

$$
\begin{aligned}
\mathbb{E}[ & h(W)-h(Z)] \\
& =\mathbb{E}[\lambda f(W+1)-W f(W)] \\
& =\mathbb{E}\left[\sum_{\alpha \in \Gamma} p_{\alpha} f(W+1)-\sum_{\alpha \in \Gamma} X_{\alpha} f(W)\right] \\
& =\sum_{\alpha \in \Gamma} \mathbb{E}\left[p_{\alpha} f(W+1)-X_{\alpha} f(W)\right] \\
& =\sum_{\alpha \in \Gamma} \mathbb{E}\left[p_{\alpha} f\left(W_{\alpha}+1\right)-X_{\alpha} f(W)\right]+\sum_{\alpha \in \Gamma} \mathbb{E}\left[p_{\alpha} f(W+1)-p_{\alpha} f\left(W_{\alpha}+1\right)\right] \\
& =\sum_{\alpha \in \Gamma} \mathbb{E}\left[p_{\alpha} f\left(W_{\alpha}+1\right)-X_{\alpha} f\left(W_{\alpha}+1\right)\right]+\sum_{\alpha \in \Gamma} \mathbb{E}\left\{p_{\alpha}\left[f(W+1)-f\left(W_{\alpha}+1\right)\right]\right\} \\
& =\sum_{\alpha \in \Gamma} \mathbb{E}\left[\left(p_{\alpha}-X_{\alpha}\right) f\left(W_{\alpha}+1\right)\right]+\sum_{\alpha \in \Gamma} \mathbb{E}\left\{p_{\alpha}\left[f(W+1)-f\left(W_{\alpha}+1\right)\right]\right\} \\
& =-\left\{\sum_{\alpha \in \Gamma} \mathbb{E}\left\{\left(X_{\alpha}-p_{\alpha}\right)\left[f\left(W_{\alpha}+1\right)-f\left(V_{\alpha}+1\right)\right]\right\}+\right. \\
& \left.\sum_{\alpha \in \Gamma} \mathbb{E}\left\{p_{\alpha} X_{\alpha}\left[f\left(W_{\alpha}+1\right)-f\left(W_{\alpha}+2\right)\right]\right\}+\sum_{\alpha \in \Gamma} \mathbb{E}\left\{\left(X_{\alpha}-p_{\alpha}\right)\left[f\left(V_{\alpha}+1\right)\right]\right\}\right\}
\end{aligned}
$$

onde na quinta e última igualdade aplicamos o Lema 1.15, parte $(a)$ e $(b)$ respectivamente. Agora, usando os demais Lemas e tomando o módulo, temos que

$$
\begin{aligned}
&|\mathbb{E}[h(W)-h(Z)]| \\
&=\mid-\left\{\sum_{\alpha \in \Gamma} \mathbb{E}\left\{\left(X_{\alpha}-p_{\alpha}\right)\left[f\left(W_{\alpha}+1\right)-f\left(V_{\alpha}+1\right)\right]\right\}+\right. \\
&\left.\sum_{\alpha \in \Gamma} \mathbb{E}\left\{p_{\alpha} X_{\alpha}\left[f\left(W_{\alpha}+1\right)-f\left(W_{\alpha}+2\right)\right]\right\}+\sum_{\alpha \in \Gamma} \mathbb{E}\left\{\left(X_{\alpha}-p_{\alpha}\right)\left[f\left(V_{\alpha}+1\right)\right]\right\}\right\} \mid
\end{aligned}
$$




$$
\begin{aligned}
& \leq\left|\sum_{\alpha \in \Gamma} \mathbb{E}\left\{\left(X_{\alpha}-p_{\alpha}\right)\left[f\left(W_{\alpha}+1\right)-f\left(V_{\alpha}+1\right)\right]\right\}\right|+ \\
& \left.\leq\left|\sum_{\alpha \in \Gamma} \mathbb{E}\left\{p_{\alpha} X_{\alpha}\left[f\left(W_{\alpha}+1\right)-f\left(W_{\alpha}+2\right)\right]\right\}\right|+\mid \sum_{\alpha \in \Gamma} \mathbb{E}\left\{\left(X_{\alpha}-p_{\alpha}\right)\left[f\left(V_{\alpha}+1\right)\right]\right\}\right\} \mid \\
& \leq\|\triangle f\| \sum_{\alpha \in \Gamma} \sum_{\substack{\beta \in B_{\alpha} \\
\beta \neq \alpha}}\left(p_{\alpha \beta}+p_{\alpha} p_{\beta}\right)+\|\Delta f\| \sum_{\alpha \in \Gamma} p_{\alpha}^{2}+b_{3}\|f\| \\
& =\|\triangle f\|\left[\sum_{\alpha \in \Gamma} \sum_{\substack{\beta \in B_{\alpha} \\
\beta \neq \alpha}} p_{\alpha} p_{\beta}+\sum_{\alpha \in \Gamma} p_{\alpha}^{2}+\sum_{\alpha \in \Gamma} \sum_{\substack{\beta \in B_{\alpha} \\
\beta \neq \alpha}} p_{\alpha \beta}\right]+b_{3}\|f\| \\
& =\|\triangle f\|\left[\sum_{\alpha \in \Gamma} \sum_{\beta \in B_{\alpha}} p_{\alpha} p_{\beta}+\sum_{\substack { \alpha \in \Gamma \\
\begin{subarray}{c}{\beta \in B_{\alpha} \\
\beta \neq \alpha{ \alpha \in \Gamma \\
\begin{subarray} { c } { \beta \in B _ { \alpha } \\
\beta \neq \alpha } }\end{subarray}} p_{\alpha \beta}\right]+b_{3}\|f\| \\
& =\|\triangle f\|\left(b_{1}+b_{2}\right)+b_{3}\|f\| \\
& \leq\left(b_{1}+b_{2}\right) \frac{1-e^{-\lambda}}{\lambda}+b_{3} \min \left(1, c_{1} \lambda^{-\frac{1}{2}}\right) .
\end{aligned}
$$

Para a segunda desigualdade usamos os Lemas 1.16, 1.17 e 1.18 , e para a última desigualdade aplicamos os Lemas 1.12 e 1.13 .

Assim, fica provada a primeira desigualdade do teorema.

Pelo Lema 1.11 temos que para $\lambda>0, \frac{1-e^{-\lambda}}{\lambda} \leq 1$. Além disso, $b_{3} \leq b_{3}^{\prime}$ e $\min \left(1, c_{1} \lambda^{-\frac{1}{2}}\right) \leq 1$. Portanto

$$
d_{V T}(W, Z) \leq b_{1}+b_{2}+b_{3}^{\prime} .
$$

Agora, da equação (1.11) temos que

$$
d_{V T}(W, Z)=\sup _{A \subset \mathbb{Z}_{+}}|\mathbb{P}(W \in A)-\mathbb{P}(Z \in A)| .
$$

Logo com $A=\{0\}$ e definindo $h_{\{0\}}(w)=1_{\{0\}}(w)-e^{-\lambda}$, temos que $\mathbb{E}[h(Z)]=0$. Aplicando Lema 1.14 segue que

$$
\begin{aligned}
\left|\mathbb{P}(W=0)-e^{-\lambda}\right| & \leq d_{V T}(W, Z) \leq\|\triangle f\|\left(b_{1}+b_{2}\right)+b_{3}\|f\| \\
& \leq\left(b_{1}+b_{2}\right) \frac{1-e^{-\lambda}}{\lambda}+b_{3} \frac{1-e^{-\lambda}}{\lambda} \\
& =\left(b_{1}+b_{2}+b_{3}\right) \frac{1-e^{-\lambda}}{\lambda} .
\end{aligned}
$$


Além disso, como $b_{3} \leq b_{3}^{\prime}$ e $\frac{1-e^{-\lambda}}{\lambda} \leq \lambda^{-1}$ para $\lambda>0$, tomando $\min \left(1, \lambda^{-1}\right)$ segue pelo Lema 1.11 que

$$
\left|\mathbb{P}(W=0)-e^{-\lambda}\right|<\min \left(1, \lambda^{-1}\right)\left(b_{1}+b_{2}+b_{3}^{\prime}\right) .
$$

Ficam assim provadas as últimas duas desigualdades. 


\section{Capítulo 2}

\section{ALGUMAS APLICAÇÕES}

No primeiro exemplo aplicamos o método de Chen-Stein ao hipercubo de dimensão $d$, com os vértices $\alpha$ correlacionados com seus vizinhos mais próximos, mas os de distância maior que 1 independentes. No segundo estudamos o bem conhecido problema dos aniversários.

Em [4], [6], [7] e [16] outros exemplos podem ser encontrados.

Exemplo 2.1: (Rinott) No hipercubo $\{0,1\}^{d}$ assuma que em cada um dos $d 2^{d-1}$ elos é definida uma direção aleatória via lançamentos independentes de uma moeda honesta e seja $W$ o número de vértices em que todos os seus $d$ elos apontam na direção deste vértice. Tomemos $\Gamma$ como o conjunto dos vértices, isto é $\Gamma=\left\{1,2, \cdots, 2^{d}\right\}$. Definamos $A$ o conjunto dos $\alpha \in \Gamma$ tal que todos os elos de $\alpha$ apontam para o vértice e seja $X_{\alpha}$ a variável indicadora definida por

$$
X_{\alpha}= \begin{cases}1, & \text { se } \alpha \in A ; \\ 0, & \text { se } \alpha \notin A .\end{cases}
$$

Segue que $W=\sum_{\alpha \in \Gamma} X_{\alpha}$ é uma variável aleatória que toma valores em $\left\{0,1, \cdots, 2^{d}\right\}$ e tem média

$$
\lambda=\mathbb{E}\left(\sum_{\alpha \in \Gamma} X_{\alpha}\right)=\sum_{\alpha \in \Gamma} \mathbb{E}\left(X_{\alpha}\right)=\sum_{\alpha \in \Gamma} p_{\alpha}
$$

onde $p_{\alpha}$ é a probabilidade de sucesso da variável $X_{\alpha}$. Como

$$
p_{\alpha}=\mathbb{P}\left(X_{\alpha}=1\right)=\frac{1}{2^{d}}
$$


então

$$
\lambda=|\Gamma| \frac{1}{2^{d}}=\frac{2^{d}}{2^{d}}=1 .
$$

Tomemos a vizinhança

$$
B_{\alpha}=\{\beta:|\alpha-\beta| \leq 1\}
$$

para $\alpha \in \Gamma$ e então $b_{2}=b_{3}^{\prime}=0$ já que para $\alpha, \beta$ vértices vizinhos

$$
p_{\alpha \beta}=\mathbb{P}\left(X_{\alpha}=1, X_{\beta}=1\right)=\mathbb{P}\left(X_{\alpha}=1\right) \mathbb{P}\left(X_{\beta}=1 / X_{\alpha}=1\right)=\frac{1}{2^{d}} \times 0=0
$$

$\mathrm{e}$

$$
\mathbb{E}\left\{X_{\alpha}-p_{\alpha} / \sigma\left(X_{\beta}: \beta \in B_{\alpha}^{c}\right)\right\}=\mathbb{E}\left(X_{\alpha}\right)-p_{\alpha}=0
$$

pois $X_{\alpha}$ e $X_{\beta}$ são independentes se $|\alpha-\beta|>1$.

Temos que $p_{\alpha}$ e $\left|B_{\alpha}\right|$ são constantes para $\alpha \in \Gamma$. Portanto

$$
b_{1}=\sum_{\alpha \in \Gamma} \sum_{\beta \in B_{\alpha}} p_{\alpha} p_{\beta}=\sum_{\alpha \in \Gamma} p_{\alpha} \sum_{\beta \in B_{\alpha}} p_{\beta}=\frac{\lambda^{2}\left|B_{\alpha}\right|}{|\Gamma|}=\frac{d+1}{2^{d}} .
$$

Segue para $Z \sim P(1)$ que

$$
d_{V T}(W, Z) \leq b_{1}+b_{2}+b_{3}^{\prime}=\frac{d+1}{2^{d}} \underset{d \rightarrow \infty}{\longrightarrow} 0 .
$$

Exemplo 2.2. (Feller [10]) (Problema dos aniversários). Como na formulação usual do problema dos aniversários, suporemos que os aniversários de $n$ indivíduos sejam independentes sobre os $d$ dias em um ano e além disso, a distribuição de probabilidade do número de nascimentos durante o ano é uniforme. Desejamos calcular a probabilidade que existam, pelo menos dois aniversários num mesmo dia. Denotemos por $W$ o número de pares de pessoas que comemoram seu aniversário no mesmo dia. A probabilidade de não haver nenhuma coincidência durante os $d$ dias do ano, ou em outras palavras, a probabilidade que todos os aniversários caiam em dias diferentes é dada para $d \geq n$ por 


$$
\begin{aligned}
p=\mathbb{P}(W=0) & =\frac{d(d-1) \cdots(d-n+1)}{d^{n}} \\
& =\frac{d}{d} \frac{d-1}{d} \cdots \frac{d-n+1}{d} \\
& =(1)\left(1-\frac{1}{d}\right) \cdots\left(1-\frac{n-1}{d}\right) \\
& =\prod_{i=1}^{n-1}\left(1-\frac{i}{d}\right) .
\end{aligned}
$$

Também,

$$
\begin{aligned}
p=\mathbb{P}(W=0) & =\frac{d(d-1) \cdots(d-n+1)}{d^{n}} \\
& =\frac{d(d-1) \cdots(d-n+1)(d-n) !}{(d-n) ! d^{n}} \\
& =\frac{d ! n !}{n !(d-n) ! d^{n}}=\left(\begin{array}{l}
d \\
n
\end{array}\right) \frac{n !}{d^{n}}
\end{aligned}
$$

onde $\left(\begin{array}{l}d \\ n\end{array}\right)$ é definido por

$$
\left(\begin{array}{l}
d \\
n
\end{array}\right)=\frac{d !}{n !(d-n) !}, \quad \text { para } d \geq n .
$$

Sabemos que para valores pequenos e positivos de $x,(0 \leq x<1), \log (1-x) \approx$ $-x$, onde que o sinal $\approx$ é usado para indicar que o quociente de um lado pelo outro converge a 1 quando $x$ vai a zero, então de (2.1) segue que

$$
\log p \approx-\frac{1+2+\cdots+(n-1)}{d}=-\frac{n(n-1)}{2 d} .
$$

Assim, admitindo que o ano tenha 365 dias temos que

$$
p=\mathbb{P}(W=0)=\prod_{i=1}^{n-1}\left(1-\frac{i}{365}\right)
$$

e verificamos que $n=23$ pessoas é o número mínimo requerido para que a probabilidade de que existam pelo menos dois aniversários num mesmo dia seja superior a 0,5 , pois

$$
p=\mathbb{P}(W=0)=0,4927<0,5,
$$


e para $n=22$ pessoas temos que

$$
p=\mathbb{P}(W=0)=0,5243,
$$

o que significa que a probabilidade que existam pelo menos dois anivesários num mesmo dia é inferior a 0.5 . Assim, para $n \geq 23$ pessoas temos uma probabilidade maior de 0.5 de obter pelo menos dois aniversários num mesmo dia.

Usando a aproximação logarítmica temos que

$$
\log p=-\frac{(23)(22)}{730}=0,69
$$

de onde $p=0,4999$.

Nosso objetivo agora é fazer uma aproximação pela distribuição Poisson e usar o Teorema 1.2 do capítulo 1 para achar uma cota superior no erro. Para isto, tomemos o conjunto $A=\{1,2, \cdots n\}$ que denota o grupo de $n$ pessoas, seja $\Gamma=\{\alpha \subset A:|\alpha|=2\}$ o conjunto índice, isto é, $\Gamma$ é o conjunto de todos os possives pares em que duas pessoas comemoram no mesmo dia seu aniversário, e seja $X_{\alpha}$ a variável aleatória com distribuição Bernoulli das pessoas indexadas por $\alpha$ que comemoram seu aniversário no mesmo dia. Então o número total de coincidências é $W=\sum_{\alpha \in \Gamma} X_{\alpha}$ e a probabilidade de sucesso da variável aleatória $X_{\alpha}$ é dada por $p_{\alpha}$ onde $W$ é uma variável aleatória que assume valores no conjunto $\left\{1,2, \cdots,\left(\begin{array}{c}n \\ 2\end{array}\right)\right\}$. Temos que

$$
p_{\alpha}=\mathbb{P}\left(X_{\alpha}=1\right)=\frac{d}{d^{2}}=\frac{1}{d} \text { para } \alpha \in \Gamma .
$$

Aproximando $W$ pela variável aleatória Poisson $Z$ com média $\lambda=\mathbb{E}(W)=$ $\mathbb{E}(Z)$, onde

$$
\lambda=\sum_{\alpha \in \Gamma} \mathbb{E}\left(X_{\alpha}\right)=\sum_{\alpha \in \Gamma} p_{\alpha}=\left(\begin{array}{l}
n \\
2
\end{array}\right) p_{\alpha}=\left(\begin{array}{l}
n \\
2
\end{array}\right) d^{-1},
$$

temos que $\mathbb{P}(W=0)$ é aproximadamente

$$
\mathbb{P}(Z=0)=e^{-\lambda}=\exp \left\{-\left(\begin{array}{l}
n \\
2
\end{array}\right) d^{-1}\right\} .
$$

Consideremos $d=365$ dias e $n=23$ pessoas, então $\lambda=\left(\begin{array}{c}23 \\ 2\end{array}\right) / 365=0,69$, logo, temos que $\mathbb{P}(Z=0)=0,4999 \geq 0,4927=\mathbb{P}(W=0)$. 
Se $\alpha \cap \beta=\emptyset$ para todo $\alpha, \beta \in \Gamma$ então $X_{\alpha}$ e $X_{\beta}$ são independentes. Tomemos a vizinhança de dependência $B_{\alpha}$ tal que $\alpha \cap \beta \neq \emptyset$. Seja então o conjunto $B_{\alpha}$ definido por

$$
B_{\alpha}=\{\beta \in \Gamma: \alpha \cap \beta \neq \emptyset\} \text {. }
$$

De onde, pela independência de $X_{\alpha}$ e $X_{\beta} \operatorname{com} \beta \in B_{\alpha}^{c}$, temos que

$$
\mathbb{E}\left\{X_{\alpha}-p_{\alpha} / \sigma\left(X_{\beta}: \beta \in B_{\alpha}^{c}\right)\right\}=\mathbb{E}\left\{X_{\alpha}-p_{\alpha}\right\}=\mathbb{E}\left(X_{\alpha}\right)-p_{\alpha}=0
$$

portanto

$$
\mathbb{E}\left|\mathbb{E}\left\{X_{\alpha}-p_{\alpha} / \sigma\left(X_{\beta}: \beta \in B_{\alpha}^{c}\right)\right\}\right|=0
$$

isto é $b_{3}=0=b_{3}^{\prime}$.

Agora vamos calcular $b_{1}, b_{2}$. Neste caso temos que $p_{\alpha}$ é constante para todo $\alpha \in \Gamma$. Temos

$$
b_{1}=\sum_{\alpha \in \Gamma} \sum_{\beta \in B_{\alpha}} p_{\alpha} p_{\beta}=|\Gamma|\left|B_{\alpha}\right| p_{\alpha}^{2}=\left(\begin{array}{l}
n \\
2
\end{array}\right)\left\{\left(\begin{array}{l}
n \\
2
\end{array}\right)-\left(\begin{array}{c}
n-2 \\
2
\end{array}\right)\right\} d^{-2}
$$

onde $\left|B_{\alpha}\right|=|\Gamma|-\left|B_{\alpha}^{c}\right|,|\Gamma|$ e $\left|B_{\alpha}\right|$ são os cardinais dos conjuntos $\Gamma$ e $B_{\alpha}$, respectivamente.

Para o cálculo de $b_{2}$, notemos que $X_{\alpha}$ e $X_{\beta}$ são independentes para todo $\alpha \neq \beta$ e portanto $p_{\alpha \beta}=p_{\alpha}^{2}$, logo

$$
\begin{aligned}
b_{2}=\sum_{\alpha \in \Gamma} \sum_{\substack{\beta \in B_{\alpha} \\
\beta \neq \alpha}} p_{\alpha \beta} & =\sum_{\alpha \in \Gamma} \sum_{\substack{\beta \in B_{\alpha} \\
\beta \neq \alpha}} p_{\alpha}^{2}=|\Gamma|\left(\left|B_{\alpha}\right|-1\right) p_{\alpha}^{2} \\
& =|\Gamma|\left|B_{\alpha}\right| p_{\alpha}^{2} \frac{\left(\left|B_{\alpha}\right|-1\right)}{\left|B_{\alpha}\right|}=b_{1} \frac{\left(\left|B_{\alpha}\right|-1\right)}{\left|B_{\alpha}\right|} .
\end{aligned}
$$

Portanto,

$$
\begin{aligned}
b_{2} & =\left(\begin{array}{l}
n \\
2
\end{array}\right)\left\{\left(\begin{array}{l}
n \\
2
\end{array}\right)-\left(\begin{array}{c}
n-2 \\
2
\end{array}\right)\right\} d^{-2} \frac{\left(\begin{array}{c}
n \\
2
\end{array}\right)-\left(\begin{array}{c}
n-2 \\
2
\end{array}\right)-1}{\left(\begin{array}{c}
n \\
2
\end{array}\right)-\left(\begin{array}{c}
n-2 \\
2
\end{array}\right)} \\
& =\left(\begin{array}{l}
n \\
2
\end{array}\right)\left\{\left(\begin{array}{l}
n \\
2
\end{array}\right)-\left(\begin{array}{c}
n-2 \\
2
\end{array}\right)-1\right\} d^{-2}
\end{aligned}
$$

e o limite superior para o erro na aproximação de $\mathbb{P}(W=0)$ por $e^{-\lambda}$ é dado por 


$$
\begin{aligned}
\mid \mathbb{P} & (W=0)-\epsilon^{-\lambda} \mid \\
& \leq\left(b_{1}+b_{2}\right) \frac{1-e^{-\lambda}}{\lambda} \\
& =\left\{\left(\begin{array}{l}
n \\
2
\end{array}\right)\left[\left(\begin{array}{l}
n \\
2
\end{array}\right)-\left(\begin{array}{c}
n-2 \\
2
\end{array}\right)\right] d^{-2}+\left(\begin{array}{l}
n \\
2
\end{array}\right)\left[\left(\begin{array}{l}
n \\
2
\end{array}\right)-\left(\begin{array}{c}
n-2 \\
2
\end{array}\right)-1\right] d^{-2}\right\} \frac{1-e^{-\lambda}}{\lambda} \\
& =\left(\begin{array}{l}
n \\
2
\end{array}\right) d^{-2}\left[2\left(\begin{array}{l}
n \\
2
\end{array}\right)-2\left(\begin{array}{c}
n-2 \\
2
\end{array}\right)-1\right] \frac{1-e^{-\lambda}}{\lambda} \\
& =\left(\begin{array}{l}
n \\
2
\end{array}\right) d^{-2}\left[2 \frac{n !}{2 !(n-2) !}-2 \frac{(n-2) !}{2 !(n-4) !}-1\right] \\
& =\left(\begin{array}{l}
n \\
2
\end{array}\right) d^{-2}[n(n-1)-(n-2)(n-3)-1] \frac{1-e^{-\lambda}}{\lambda} \\
& =\left(\begin{array}{l}
n \\
2
\end{array}\right) d^{-2}\left[n^{2}-n-n^{2}+5 n-6-1\right] \frac{1-e^{-\lambda}}{\lambda} \\
& =\frac{1}{d^{2}}\left(\begin{array}{l}
n \\
2
\end{array}\right)(4 n-7) \frac{1-e^{-\lambda}}{\lambda} \\
& =\frac{1}{d}(4 n-7)\left(1-e^{-\lambda}\right) \leq \frac{4 n}{d} .
\end{aligned}
$$

Tomando $d$ da ordem de $n^{2}$ temos,

$$
\left|\mathbb{P}(W=0)-e^{-\lambda}\right| \leq \frac{c^{\prime}}{n} \underset{n \rightarrow \infty}{\longrightarrow} 0
$$

onde $c^{\prime}>0$ uma constante independente de $n$. 


\section{Capítulo 3}

\section{MODELO DE EHRENFEST}

P. Ehrenfest e T. Ehrenfest, (1907) [9], introduziram um modelo de bolas e urnas ilustrando a conexão entre as dinâmicas de sistemas microscópicos e macroscópicos. Especificamente mostraram que uma evolução macroscópica irreversível não é, em si, incompatível com a reversibilidade do sistema microscópico, estudando também seus tempos de recorrência.

Suponhamos duas urnas $U_{1}, U_{2}$ e $N$ bolas, numeradas de 1 a $N$, distribuídas ao acaso entre as urnas. A cada unidade de tempo é escolhido um número aleatório em $\{1,2, \cdots, N\}$ (distribuição uniforme em $\{1,2, \cdots, N\}$, sendo todos os sorteios independentes). A bola com o número sorteado será trocada de urna. Este processo será repetido ao longo dos instantes de tempos $\{0,1,2, \ldots\}$.

O modelo de bolas e urnas de Ehrenfest é conhecido como um modelo de difusão de partículas entre dois recipientes, se identificamos as bolas com partículas e assumimos que as duas urnas representam agora compartimentos de mesmo volume, que contenham um "gás ideal" separado por uma membrana que permite o trânsito de um compartimento a outro. Assim as $N$ partículas do gás (que é algo da ordem de $10^{23}$ ) estão distribuídas nos dois compartimentos e nossa variável de interesse será o número de partículas que se encontram em cada instante em um determinado recipiente.

A evolução do gás entre os dois compartimentos pode ser visto como um passeio aleatório no hipercubo. Trataremos então o modelo de Ehrenfest no contexto do passeio aleatório no hipercubo e a nossa variável aleatória de interesse 
será o número de visitas que o passeio faz (eventualmente) à origem. Em termos do modelo de bolas e urnas será o número de vezes que uma urna previamente escolhida fica vazia durante o processo.

Denotaremos por $\Omega_{N}=\{0,1\}^{N}$ o conjunto de configurações. Os elementos de $\Omega_{N}$ serão denotados por $\xi=\left(\xi_{1}, \ldots, \xi_{N}\right) \in\{0,1\}^{N}$ onde $\xi_{i} \in\{0,1\}$ para todo $i \in\{1, \ldots, N\}$. Seus vizinhos são todos os elementos que tem todas as coordenadas menos uma, iguais às coordenadas de $\xi$. Definiremos a distância entre as configurações $\xi$ e $\zeta$ por

$$
|\xi-\zeta|=\sum_{i=1}^{N}\left|\xi_{i}-\zeta_{i}\right|
$$

A distância entre $\xi$ e $\zeta$ é o número de coordenadas onde $\xi$ e $\zeta$ diferem. Dois pontos de $\Omega_{N}$ são vizinhos se $|\xi-\zeta|=1$.

Para o passeio aleatório $\xi(t)$ em $\Omega_{N}$, tomemos $\xi(0)=\eta, \eta \in \Omega_{N}$ e consideremos a seguinte evolução estocástica em $\Omega_{N}$ : dada a configuração $\xi(t)$ no tempo $t$, após um tempo aleatório, a configuração decide mudar uma de suas componentes com probabilidade 1. Quando decide mudar, ela salta para uma posição vizinha escolhida ao acaso, com distribuição uniforme (com probabilidade $\frac{1}{N}$ ); aqui a palavra saltar será utilizada para referir que uma configuração $\xi$ passa à configuração $\xi^{\prime}$, onde $\xi$ e $\xi^{\prime}$ diferem numa coordenada. Em $\Omega_{N}$ temos que a medida $\nu(\xi)=2^{-N}$, para toda $\xi \in \Omega_{N}$ é invariante. Observe que o passeio aleatório é periódico.

Vamos definir $Y^{\nu}(\alpha)=N-\sum_{i=1}^{N} \xi_{i}^{\nu}(\alpha)$ como o número de zeros na configuração $\xi$ após o $\alpha$-ésimo salto iniciado segundo a configuração escolhida no suporte de $\nu$. Seja $Y^{\nu}(\alpha)$ a configuração no instante $\alpha$ que iniciou segundo a medida invariante, isto é, com

$$
Y^{\nu}(0)=\sum_{\eta \in \Omega_{N}} \nu(\eta) Y^{\eta}(0)=\sum_{\eta \in \Omega_{N}} \frac{Y^{\eta}(0)}{2^{N}}
$$

Como as escolhas dos vértices são feitas de maneira independente temos que $\left\{Y^{\nu}(\alpha): \alpha \geq 0\right\}$ forma uma cadeia de Markov com espaço de estados $S=$ $\{0, \ldots, N\}$ e probabilidade de transição para $i, j \in S$ dada por 


$$
p_{i, j}=\left\{\begin{array}{lll}
\frac{N-i}{N}, & \text { se } j=i+1 & \text { e } i=0,1, \ldots, N-1 \\
\frac{i}{N}, & \text { se } j=i-1 & \text { e } i=1,2, \ldots, N \\
0, & \text { caso contrário. } &
\end{array}\right.
$$

Para calcular os valores de $b_{1}, b_{2}, b_{3}\left(b_{3}^{\prime}\right)$ do método de Chen-Stein no modelo de Ehrenfest, utilizaremos um lema (ver [13], Lema I, pág 13) e um resultado dado por Aldous (ver [1], resultado 2.7).

Definamos agora a seqüência de variáveis aleatórias $I(t)$ com $t=1,2, \ldots$, assumindo valores no conjunto $\{1, \ldots, N\}$ independentes e identicamente distribuidas com $\mathbb{P}\{I(t)=k\}=\frac{1}{N}$ para $k \in\{1,2, \ldots, N\}$. O Lema de [13] pode ser escrito como:

Lema 3.1 A probabilidade do primeiro retorno do passeio aleatório $\xi(t)$, a uma posição já visitada em 6 ou mais saltos, é menor ou igual a $\frac{8}{N^{3}}$. (A. Galves $\epsilon$ C. Peixoto [13]).

Agora. consideremos $X_{\alpha}=1_{\left\{Y^{\nu}(\alpha)\right\}}(N)$ variável Bernoulli, isto é,

$$
X_{\alpha}= \begin{cases}1, & \text { se o passeio está no origem após o } \alpha \text {-ésimo salto } \\ 0, & \text { caso contrário }\end{cases}
$$

e tomemos $\Gamma=\left\{0, \cdots, 2^{N} t\right\}$ e seja $W=\sum_{\alpha \in \Gamma} X_{\alpha}$, com $\alpha \in \Gamma$ e $W$ o número de vezes que o passeio visita o origem, isto é, o número de vezes em que todas as componentes da configuração $\xi$ são zeros, $t>0$. O valor médio de $W$ é dado por

$$
\mathbb{E}(W)=\mathbb{E}\left(\sum_{\alpha \in \Gamma} X_{\alpha}\right)=\sum_{\alpha \in \Gamma} \mathbb{E} X_{\alpha}=\sum_{\alpha \in \Gamma} p_{\alpha}=|\Gamma| \nu=t
$$

onde $p_{\alpha}=\nu=2^{-N}$.

Seja $B_{\alpha}=\left\{\beta \in \Gamma:|\alpha-\beta|<N^{2}\right\}$, o conjunto vizinhança de $\alpha$. Calculando os valores do método de Chen-Stein temos

$$
b_{1}=\sum_{\alpha \in \Gamma} \sum_{\beta \in B_{\alpha}} p_{\alpha} p_{\beta}=|\Gamma|\left|B_{\alpha}\right| p_{\alpha}^{2}=|\Gamma|\left(2 N^{2}+1\right) \nu^{2}=\frac{|\Gamma|\left(2 N^{2}+1\right)}{2^{2 N}} .
$$


Pelo Lema 3.1 temos que a probabilidade de retorno a uma posição já visitada em 6 ou mais saltos é menor ou igual a $\frac{8}{N^{3}}$, isto é

$$
\mathbb{P}\left(X_{\alpha+2 k}=1 / X_{\alpha}=1\right) \leq \frac{8}{N^{3}}, \quad \text { para todo } k \geq 3 .
$$

Dado que o passeio está no origem, resta determinar as probabilidades de retornar em 2 e 4 saltos respectivamente.

Para retornar ao origem em 2 saltos temos que

$$
\mathbb{P}\left(X_{\alpha+2}=1 / X_{\alpha}=1\right)=\frac{1}{N},
$$

e para retornar em 4 saltos

$$
\mathbb{P}\left(X_{\alpha+4}=1 / X_{\alpha}=1\right)=\frac{2(N-1)}{N^{3}}+\frac{1}{N^{2}} \leq \frac{2}{N^{2}}+\frac{1}{N^{2}}=\frac{3}{N^{2}} .
$$

Calculando $b_{2}$;

$$
\begin{aligned}
& b_{2}=\sum_{\alpha \in \Gamma} \sum_{\substack{\alpha \in B_{\alpha} \\
\beta \neq \alpha}} p_{\alpha \beta}=\sum_{\alpha \in \Gamma} \sum_{\substack{\beta \in B_{\alpha} \\
\beta \neq \alpha}} \mathbb{E}\left(X_{\alpha} X_{\beta}\right)=\sum_{\alpha \in \Gamma} \sum_{\substack{\beta \in B_{\alpha} \\
\beta \neq \alpha}} \mathbb{P}\left(X_{\alpha}=1, X_{\beta}=1\right) \\
& =\sum_{\alpha \in \Gamma}\left[\sum_{\substack{\beta \in B_{\alpha} \\
\beta>\alpha \\
\beta \neq \alpha}} \mathbb{P}\left(X_{\alpha}=1\right) \mathbb{P}\left(X_{\beta}=1 / X_{\alpha}=1\right)\right. \\
& \left.+\sum_{\substack{\beta \in \alpha_{\alpha} \\
\text { a子a } \\
\beta \neq \alpha}} \mathbb{P}\left(X_{\beta}=1\right) \mathbb{P}\left(X_{\alpha}=1 / X_{\beta}=1\right)\right] \\
& =\sum_{\alpha \in \Gamma}\left[2 \nu \sum_{l=1}^{\left|\frac{N^{2}}{4}\right|} \mathbb{P}\left(X_{\alpha+2 l}=1 / X_{\alpha}=1\right)\right] \\
& \leq 2 \nu|\Gamma|\left(\frac{1}{N}+\frac{3}{N^{2}}+\frac{8 N^{2}}{4 N^{3}}\right)=2 \nu|\Gamma|\left(\frac{1}{N}+\frac{3}{N^{2}}+\frac{2}{N}\right) \\
& =2 \nu|\Gamma|\left(\frac{3}{N}+\frac{3}{N^{2}}\right) \leq 2 \nu|\Gamma|\left(\frac{3}{N}+\frac{3}{N}\right)=\frac{12 \nu|\Gamma|}{N}=\frac{12|\Gamma|}{2^{N} N} .
\end{aligned}
$$

Finalmente, Aldous (1983) mostrou que para todo $t \geq N^{2}$ e todas $\xi, \zeta \in \Omega_{N}$,

$$
\left|p_{\xi, \zeta}(t)-\frac{1}{2^{N}}\right| \leq \frac{1}{2^{2 N}}
$$


onde $p_{\xi, \zeta}(t)$ é a probabilidade de transição do passeio passar da configuração $\xi$ à configuração $\zeta$ no tempo $t$.

Como $p_{0, \xi}(t)=p_{\xi, Q}(t)$ para todo $t \geq 0$ e $\underset{\sim}{0}$ é a configuração onde todas suas componentes são zeros, segue que

$$
\begin{aligned}
\sum_{\alpha \in \Gamma} \mathbb{E}\left|\mathbb{E}\left\{X_{\alpha}-p_{\alpha} / \sigma\left(X_{\beta}: \beta \in B_{\alpha}^{c}\right)\right\}\right| & \leq \sum_{\alpha \in \Gamma} \mathbb{E}\left|\mathbb{E}\left\{X_{\alpha}-p_{\alpha} / \sigma\left(\xi_{\beta}^{\nu}: \beta \in B_{\alpha}^{c}\right)\right\}\right| \\
& =\sum_{\alpha \in \Gamma} \sum_{\xi \in \Omega_{N}}\left|\mathbb{E}\left\{X_{\alpha} / \xi_{\alpha+N^{2}}^{\nu}=\xi\right\}-p_{\alpha}\right| \nu(\xi) \\
& =\sum_{\alpha \in \Gamma} \sum_{\xi \in \Omega_{N}}\left|p_{\xi, 0}\left(N^{2}\right)-p_{\alpha}\right| \nu(\xi) \\
& \leq \sum_{\alpha \in \Gamma} \nu^{2}=|\Gamma| \nu^{2}=\frac{|\Gamma|}{2^{2 N}} .
\end{aligned}
$$

Concluímos então

$$
\begin{aligned}
d_{V T}(W, Z) & \leq \frac{|\Gamma|\left(2 N^{2}+1\right)}{2^{2 N}}+\frac{12|\Gamma|}{2^{N} N}+\frac{|\Gamma|}{2^{2 N}} \\
& =\frac{|\Gamma|}{N}\left[\frac{N\left(2 N^{2}+2\right)}{2^{2 N}}+\frac{12}{2^{N}}\right] .
\end{aligned}
$$

Portanto

$$
\begin{aligned}
d_{V T}(W, Z) & \leq \frac{2^{N} t}{N}\left[\frac{N\left(2 N^{2}+2\right)}{2^{2 N}}+\frac{12}{2^{N}}\right] \\
& =\frac{t}{N}\left[\frac{N\left(2 N^{2}+2\right)}{2^{N}}+12\right] \\
& =\frac{c_{2} t}{N} \underset{N \rightarrow \infty}{\longrightarrow} 0, \text { para todo } t>0 .
\end{aligned}
$$




\section{BIBLIOGRAFIA}

[1] D. Aldous, Minimization Algorithms and Random Walk on the d-Cube, Ann. Probab, vol 11(2), 403 - 413, 1983.

[2] D. Aldous, Probability Approximations Via the Poisson Clumping Heuristic, Springer, Berlin, 1989.

[3] R. Arratia, L. Goldstein and L. Gordon, Poisson Approximation and the ChenStein Method, Stat. Science, vol 5(4), 403 - 434, 1990.

[4] R. Arratia, L. Goldstein and L. Gordon, Two Moments suffice for Poisson Approximations: The Chen-Stein method, Ann. Probab, vol 17(1), $9-25$, 1989.

[5] R. Arratia and S. Tavare, Book Review: Review of Probability Approximations Via de Poisson Clumping Heuristic by D. Aldous and Poisson Approximations by A. D. Barbour, L. Holst And S. Janson, Ann. Praobab, vol 21(4), 2269 - 2279, 1993.

[6] A. D. Barbour and G. K. Eagleson, Poisson approximation for some statistics based on exchangeable trials, Adv. in Appl. Probab, vol 15, 585 - 600, 1983.

[7] A. D. Barbour, L. Holst and S. Janson, Poisson Approximation, Oxford, University Press, New York, 1992.

[8] R. Durrett, Probability: Theory and Examples, California, 1991.

[9] P. Ehrenfest and T. Ehrenfest, Über zwei bekannte Einwände gegen das Boltzmannsche H-Theorem, Physikalische Zeitschrift, vol 8, 311 - 314, 1907. Para uma discussão em termos matemáticos veja M. Kac, Random Walk 
and the Theory of Brownian. Motion, Amer. Math. Monthly, vol 54, $369-$ 391, 1947.

[10] W. Feller, An Introduction to Probability Theory and Its Applications, vol I. New York, London, Sydney, Third edition, 1968.

[11] P. A. Ferrari e A. Galves, Acoplamento e Processos Estocásticos, Brasil, 21ํㅡㄴ Colóquio Brasileiro de Matemática, 1997.

[12] A. Galves, A. C. R. Nogueira e M. E. Vares, Introdução aos Sistemas Markovianos de Partículas, Brasil, 5o Simpósio Nacional de Probabilidade e Estatística. 1982.

[13] C. Peixoto, Aproximaçăo do Equilíbrio e Tempos Exponenciais para o Passeio Aleatório no Hipercubo, Tese de Mestrado, Universidade de São Paulo, Brasil, 1992.

[14] C. M. Stein, Approximate Computation of Expectations, vol 7, IMS, Hayward, California, 1986.

[15] C. M. Stein, A bound for the error in the normal approximation to the distribution of a sum of dependent random variables, Proc. Sixth Berkeley Symp. Math. Statist. Probab, vol 2, 583 - 602, 1970.

[16] Louis H. Y. Chen, Poisson Approximation for Dependent trials, Ann. Probab, vol 3, $534-545,1975$. 